\title{
The DeoR-type transcriptional regulator SugR acts as a repressor for genes encoding the phosphoenolpyruvate:sugar phosphotransferase system (PTS) in Corynebacterium glutamicum
}

\author{
Lars Gaigalat ${ }^{1,2}$, Jan-Philip Schlüter ${ }^{1,2}$, Michelle Hartmann ${ }^{1,2,3}$, \\ Sascha Mormann 1,2,4, Andreas Tauch ${ }^{1}$, Alfred Pühler ${ }^{2}$ and Jörn Kalinowski*1
}

Address: ${ }^{1}$ Institut für Genomforschung, Universität Bielefeld, D-33594 Bielefeld, Germany, ${ }^{2}$ Lehrstuhl für Genetik, Universität Bielefeld, D-33594 Bielefeld, Germany, ${ }^{3}$ ISAS - Institute for Analytical Sciences, Department of Metabolomics, Bunsen-Kirchhoff-Str. 11, 44139 Dortmund, Germany and ${ }^{4}$ University of Applied Sciences Lippe and Hoexter, Life Science Technologies, Laboratory of Microbiology, Liebigstr. 87, 32657 Lemgo, Germany

Email: Lars Gaigalat - lars.gaigalat@Genetik.Uni-Bielefeld.DE; Jan-Philip Schlüter - jschluet@Genetik.Uni-Bielefeld.DE; Michelle Hartmann - hartmann@isas.de; Sascha Mormann - smormann@Genetik.Uni-Bielefeld.DE; Andreas Tauch - tauch@Genetik.UniBielefeld.DE; Alfred Pühler - puehler@Genetik.Uni-Bielefeld.DE; Jörn Kalinowski* - Joern.Kalinowski@Genetik.Uni-Bielefeld.DE

* Corresponding author

Published: 15 November 2007

BMC Molecular Biology 2007, 8:104 doi:10.1 186/147|-2199-8-104
Received: 28 February 2007

Accepted: 15 November 2007

This article is available from: http://www.biomedcentral.com/I47I-2/99/8/104

(C) 2007 Gaigalat et al; licensee BioMed Central Ltd.

This is an Open Access article distributed under the terms of the Creative Commons Attribution License (http://creativecommons.org/licenses/by/2.0), which permits unrestricted use, distribution, and reproduction in any medium, provided the original work is properly cited.

\begin{abstract}
Background: The major uptake system responsible for the transport of fructose, glucose, and sucrose in Corynebacterium glutamicum ATCC 13032 is the phosphoenolpyruvate:sugar phosphotransferase system (PTS). The genes encoding PTS components, namely ptsl, pts $\mathrm{H}$, and ptsF belong to the fructose-PTS gene cluster, whereas $p t s G$ and $p t s S$ are located in two separate regions of the $C$. glutamicum genome. Due to the localization within and adjacent to the fructose-PTS gene cluster, two genes coding for DeoR-type transcriptional regulators, cg2 II 8 and sugR, are putative candidates involved in the transcriptional regulation of the fructose-PTS cluster genes.
\end{abstract}

Results: Four transcripts of the extended fructose-PTS gene cluster that comprise the genes sugRcg2 I /6, ptsl, cg2 I / 8-fruK-ptsF, and pts $\mathrm{H}$, respectively, were characterized. In addition, it was shown that transcription of the fructose-PTS gene cluster is enhanced during growth on glucose or fructose when compared to acetate. Subsequently, the two genes sugR and $\operatorname{cg} 21 / 8$ encoding for DeoR-type regulators were mutated and PTS gene transcription was found to be strongly enhanced in the presence of acetate only in the sugR deletion mutant. The SugR regulon was further characterized by microarray hybridizations using the $\operatorname{sug} R$ mutant and its parental strain, revealing that also the PTS genes pts $G$ and ptsS belong to this regulon. Binding of purified SugR repressor protein to a 21 bp sequence identified the SugR binding site as an AC-rich motif. The two experimentally identified SugR binding sites in the fructose-PTS gene cluster are located within or downstream of the mapped promoters, typical for transcriptional repressors. Effector studies using electrophoretic mobility shift assays (EMSA) revealed the fructose PTS-specific metabolite fructose-I-phosphate (F-I-P) as a highly efficient, negative effector of the SugR repressor, acting in the micromolar range. Beside F-I-P, other sugar-phosphates like fructose-I,6-bisphosphate (F-I,6P) and glucose-6-phosphate (G-6-P) also negatively affect SugR-binding, but in millimolar concentrations. 


\begin{abstract}
Conclusion: In C. glutamicum ATCC I3032 the DeoR-type regulator SugR acts as a pleiotropic transcriptional repressor of all described PTS genes. Thus, in contrast to most DeoR-type repressors described, SugR is able to act also on the transcription of the distantly located genes ptsG and ptsS of C. glutamicum. Transcriptional repression of the fructose-PTS gene cluster is observed during growth on acetate and transcription is derepressed in the presence of the PTS sugars glucose and fructose. This derepression of the fructose-PTS gene cluster is mainly modulated by the negative effector F-I-P, but reduced sensitivity to the other effectors, F-I,6-P or G-6-P might cause differential transcriptional regulation of genes of the general part of the PTS (ptsl, $p t s H)$ and associated genes encoding sugar-specific functions (ptsF, ptsG, ptsS).
\end{abstract}

\section{Background}

A sugar transport system which is widespread among various microorganisms is the phosphoenolpyruvate:sugar phosphotransferase system (PTS) $[1,2]$. The PTS is characterized by the uptake of the carbon source, which is simultaneously phosphorylated resulting in intracellular sugarphosphate. The transfer of the phosphate group to its substrate consists of five distinct reactions. The first step is the autophosphorylation of enzymeI, where phosphoenolpyruvate acts as the phosphate donor. Secondly, enzymeI transfers the phosphate group to the His-15 residue of the HPr protein. EnzymeI and HPr together are designated the general or central part of the PTS. HPr, in turn, transfers the phosphate group to the substrate-specific enzymesII. In general, enzymeII consists of two hydrophilic domains (IIA and IIB) and one transmembrane domain (IIC). Subsequently, the phosphate group is transferred within the enzymeII complex from domain IIA to domain IIB. The IIC-domain transports the substrate into the cell and the phosphate group is finally transfered from IIB to the substrate and the phosphorylated sugar is released into the cytoplasm [3].

The fast growing, Gram-positive bacterium Corynebacterium glutamicum is widely used for the fermentative production of amino acids [4] and the PTS system is the major uptake system responsible for the transport of fructose, glucose, and sucrose in this organism $[5,6]$. In $C$. glutamicum ATCC 13032 five genes were identified, which encode proteins with known functions in the PTS (Table 1). In detail, ptsI encodes the enzymeI and $p t s H$ the enzyme HPr of the general part of the PTS [7]. Furthermore, $p t s G$ encodes the sugar-specific enzymeII involved in the uptake of glucose, ptsS is involved in sucrose uptake, and finally $p t s F$ is responsible for the uptake of fructose $[7,8]$. However, physiological results were already obtained for the function of all PTS genes with known function in C. glutamicum [7,9]. Additionally, for the general part of the PTS, the activities of enzymeI and HPr were demonstrated in a cell-free assay. PTS-dependent transport and the physiological function of the pts $G$ gene in the uptake of glucose were also investigated $[5,10]$. Genetically engineered mutations in the putative glucose- and sucrose-specific enzymesII encoded by $p t s G$ and ptsS, revealed impaired growth on glucose and sucrose, respectively, indicating glucose transport by PtsG and sucrose transport by PtsS [8]. Inactivation of the $p t s F$ gene resulted in a significantly reduced growth on fructose as a sole carbon source, therewith indicating the function of PtsF in fructose uptake [6,8]. Furthermore, PtsF-defective mutants showed a diminished growth rate compared to the wild type with sucrose as a sole carbon source, and additionally an accumulation of external fructose during exponential growth [6]. Due to the lack of a fructokinase activity in C. glutamicum the internally liberated fructose, resulting from the cleavage of sucrose-6-phosphate taken up by PtsS, cannot be metabolized and is exported $[11,8]$. Therefore, PtsF activity is also necessary to import the external fructose in order to completely metabolize sucrose. Furthermore, Moon et al. [8] identified one enzymeII encoded by cg3365 and cg3366 with unknown substrate specifity.

The transcriptional regulation of genes involved in PTS transport are well studied in a variety of Gram-negative and low-G+C Gram-positive bacteria [12-15]. However, little is known about gene regulation of PTS in Corynebacteria. An HPr-kinase/phosphatase activity as well as the essentially conserved serine-residue 46 in the HPr amino acid sequence, which exerts carbon catabolite control in low-G+C Gram-positive bacteria, was not detected in $C$. glutamicum $[7,16]$. Additionally, genes encoding transcriptional antiterminators of the BglG-family like GlcT of B. subtilis (regulation of the ptsGHI operon) or BglG of $E$. coli (regulation of the $\beta$-glucoside PTS) are only represented by a pseudogene in the genome of $C$. glutamicum ATCC 13032 (cg3144'/NCgl2743') [17]. However, it has to be noted that other C. glutamicum isolates apparently have functional copies of antiterminator-like genes as well as additional genes for enzymes II [18]. As another transcriptional regulatory system, in the low G+C Gram-positive bacterium Streptococcus gordonii the DeoR-type regulator FruR was characterized, which controls fructosePTS gene expression in dependence of fructose, sucrose or xylitol [15]. The expression of the PTS genes $p t s F, p t s G$, and $p t s S$ in C. glutamicum was initially considered to be consti- 
Table I: Molecular characteristics of PTS and putative PTS associated genes of $C$. glutamicum ATCCI3032

\begin{tabular}{|c|c|c|c|c|c|c|}
\hline & CDS & NCBI No. ${ }^{2}$ & gene name & size [aa] & size $[\mathrm{kDa}]$ & Annotation \\
\hline \multirow[t]{2}{*}{ ext. } & $\operatorname{cg} 21115$ & NCgII856 & $\operatorname{sug} R$ & 259 & 27.6 & $\begin{array}{l}\text { Transcriptional regulator protein, DeoR- } \\
\text { family }\end{array}$ \\
\hline & $\operatorname{cg} 2116$ & $\mathrm{NCg} \mid \mathrm{I} 857$ & - & 320 & 34.1 & Putative I-phosphofructokinase \\
\hline \multirow[t]{5}{*}{ fructose-PTS } & $\operatorname{cg} 2117$ & $\mathrm{NCg} \mid \mathrm{l} 858$ & ptsl & 568 & 59.6 & $\begin{array}{l}\text { Phosphotransferase system (PTS), enzyme } \\
\text { I }\end{array}$ \\
\hline & $\operatorname{cg} 2118$ & NCgl| 859 & - & 264 & 28.0 & $\begin{array}{l}\text { Putative transcriptional regulator protein, } \\
\text { DeoR-family }\end{array}$ \\
\hline & $\operatorname{cg} 2119$ & NCgII 860 & fruK & 330 & 34.0 & I-phosphofructokinase \\
\hline & $\operatorname{cg} 2 / 20$ & $\mathrm{NCgl|86I}$ & $p t s F$ & 688 & 70.5 & $\begin{array}{l}\text { Fructose-specific PTS component, enzyme } \\
\text { IIABC }\end{array}$ \\
\hline & $\operatorname{cg} 2|2|$ & NCgII 862 & ptsH & 89 & 9.1 & $\begin{array}{l}\text { Phosphocarrier protein HPr, PTS } \\
\text { component }\end{array}$ \\
\hline \multirow[t]{4}{*}{ non clustered } & $\operatorname{cg} / 537$ & NCgl| 305 & $p t s G$ & 683 & 72.6 & $\begin{array}{l}\text { Glucose-specific PTS component, enzyme } \\
\text { IIBCA }\end{array}$ \\
\hline & $\operatorname{cg} 2925$ & NCgl2553 & ptsS & 661 & 69.1 & $\begin{array}{l}\text { Sucrose-specific PTS component, enzyme } \\
\text { IIBCA }\end{array}$ \\
\hline & $\operatorname{cg} 3365$ & NCgl2933 & - & 513 & 52.7 & $\begin{array}{l}\text { L-ascorbate type PTS component, enzyme } \\
\text { IIC }\end{array}$ \\
\hline & $\operatorname{cg} 3366$ & NCgl2934 & - & 270 & 29.0 & $\begin{array}{l}\text { L-ascorbate type PTS component, enzyme } \\
\text { IIAB }\end{array}$ \\
\hline
\end{tabular}

I Coding sequences (CDS) are numbered in reference to the complete genome sequence of $C$. glutamicum (GenBank accession number BX927I47).

2 Numbers in the genome database of the National Center for Biotechnology Information (NCBI No.).

tutive [19], but recently, ptsG transcription was observed to be induced by switching from biomass to L-lysine production [20]. However, a global carbon regulation in Corynebacterium glutamicum could not be observed until now, but candidate genes associated with the PTS were recently reviewed [21].

The aim of this study was the characterization of the transcriptional regulation of PTS genes in C. glutamicum. By first analysing gene transcription in the fructose-PTS gene cluster and transcriptional regulation of all known PTS genes, we found a PTS-sugar specific transcriptional regulation of all PTS genes. By deletion mutagenesis of $c g 2115$ $(\operatorname{sugR})$ and expression analysis by real-time-RT-PCR and microarray hybridization, SugR was identified as a pleiotropic transcriptional regulator of all PTS genes. Electrophoretic mobility shift assays with purified SugR protein were employed to determine the SugR binding sites and its effector metabolites.

During the preparation of this manuscript, Engels and Wendisch [22] reported the identification of the DeoRtype transcriptional regulator encoded by the gene sugR in C. glutamicum, acting as a transcriptional repressor of the ptsG gene. By comparing a sugR mutant with its parent strain by microarray hybridization, these authors found that the $p t s F$ and $p t s S$ genes are under the control of this regulator, which they termed SugR. The transcriptional role of SugR was extended in this work to the fructose-PTS cluster genes ptsI, cg2118, fruK, and ptsH and completes the understanding for the expression of all described PTS genes in C. glutamicum in general and the expression of the genes of the fructose-PTS cluster in detail. Furthermore, the effector studies in this work points to a sugar specific regulation of these genes similar to the TrmB regulator of Thermococcus litoralis and Pyrococcus furiosus $[23,24]$.

\section{Results}

The extended fructose-PTS gene cluster of C. glutamicum contains four transcriptional units

In the genome of C. glutamicum ATCC 13032 [17], the locations of the five PTS genes $p t s I, p t s H, p t s F, p t s G$, and $p t s S$ were identified. On this account, the PTS genes can be grouped into the fructose-PTS gene cluster (ptsI, cg2118, fruK, $p t s F$, and $p t s H$ ) and the distantly located glucose- and sucrose-PTS genes $p t s G$ and $p t s S$, respectively, as well as the hitherto uncharacterized genes co3365 and cg3366 (Table 1 ). The genes ptsI, cg2118, fruK, ptsF, and ptsH of the fructose-PTS cluster together with the region encoding sugR and $c g 2116$ form the extended fructose-PTS gene cluster (Table 1).

In order to analyse the transcriptional organization of the extended fructose-PTS gene cluster, mRNA transcripts overlapping adjacent coding regions were investigated by real-time RT-PCR. In these experiments, mRNAs spanning the intergenic regions between $\operatorname{sugR}$ and $c g 2116, c g 2118$ and $f r u K$, fruK and $p t s F$, and between $p t s F$ and $p t s H$, respectively, were detected in the C. glutamicum strain RES167 grown on glucose (data not shown). However, a non-coding region of $188 \mathrm{bp}$ between the coding regions of $p t s F$ 
and $p t s H$ was observed indicating a possible promoter upstream of $p t s H$. Considering these observations and the localization of $p t s I$ on the opposite strand, it is most likely, that the genes $s u g R$-cg2116 and $c g 2118$-fruK-ptsF form two operons, whereas ptsI is monocistronic. Although a readthrough transcription from $p t s F$ to $p t s H$ was detected, the $p t s H$ gene is probably also transcribed monocistronically. The operon predictions by the computer program VIMSS [25] and the predicted positions of transcriptional terminators 3' of cg2116, ptsI and ptsH determined by the computer program TransTerm [26], depicted as stem-loop structures in Fig. 1A, support this view.

Consistent with these interpretations, four transcriptional start sites were localized upstream from the genes $s u g R$, ptsI, cg2118, and ptsH, using the rapid amplification of cDNA ends (RACE) method (Fig. 1). The deduced promoter sequences showed similarities to the sequences of the -10 (Tac/taaT) and -35 (TTTGCC/TTGGCA/TTGCCA) regions of the C. glutamicum consensus promoter [27]. The promoter of the cg2118-fruK-ptsF operon showed the highest similarity to the consensus promoter, whereas the promoters of sugR-cg2116, ptsI, and ptsH were less conserved, especially in the -35 region, (Fig. 1B). Further- more, it was found that the divergent promoters $\mathrm{P}_{p t s I}$ and $\mathrm{P}_{c g 2118}$ produce mRNAs overlapping by a complementary sequence of 14 nucleotides.

Transcription of the fructose-PTS gene cluster is enhanced in C. glutamicum cultures grown on glucose or fructose when compared to cultures grown on acetate

In order to investigate a carbon source-dependent expression of the genes belonging to the extended fructose-PTS gene cluster, the transcription levels were investigated by RT-PCR experiments. For this purpose, total mRNA was isolated from C. glutamicum RES167, grown in minimal media containing acetate, glucose or fructose as a sole carbon source. Glucose and fructose were employed as PTS sugars requiring the expression of the PTS gene cluster for their uptake, whereas acetate represents a non-PTS carbon source. The relative mRNA amounts of the genes belonging to the extended fructose-PTS cluster (sugR to ptsH) were determined using real-time RT-PCR. The values from the glucose- and fructose-grown cultures were compared to those obtained from cultures grown on acetate (Fig. 2). The mRNA levels of $s u g R$ and $c g 2116$ showed only minor changes during growth on glucose and fructose in comparison to acetate. In contrast to this, all genes of the fruc-

A)

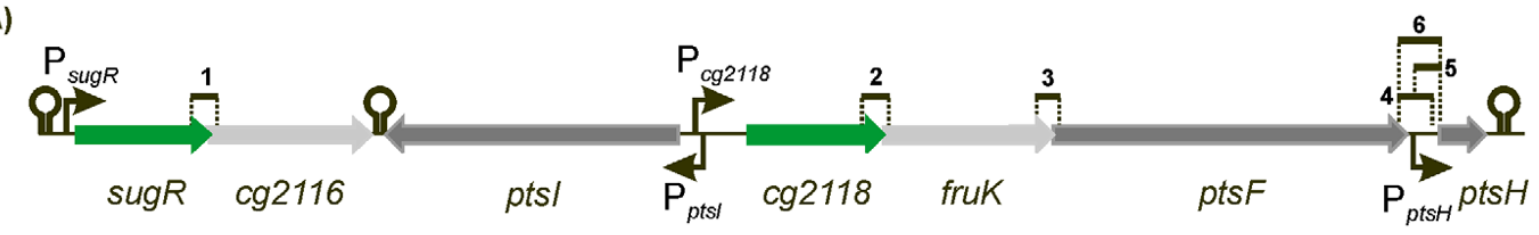

B)

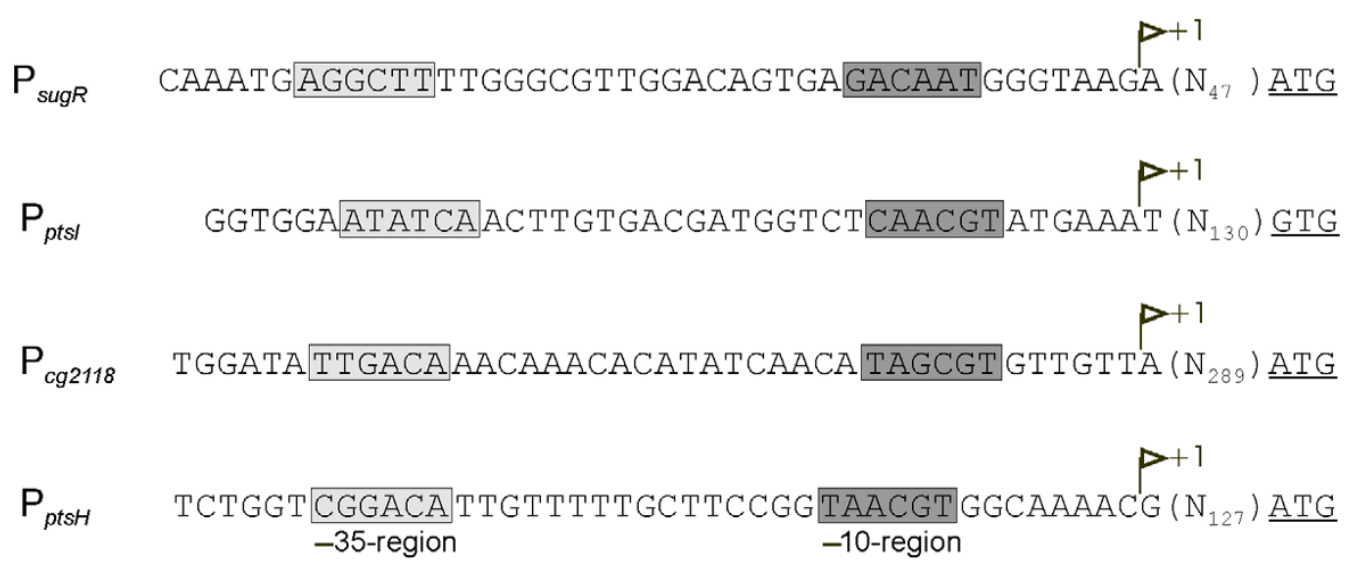

\section{Figure I}

The transcriptional organization of the genes in the extended fructose-PTS cluster of $C$. glutamicum. A) The extended fructosePTS gene cluster as well as its transcriptional elements are depicted. Gene overlapping RT-PCR products are indicated by black bars and numbered. Transcriptional terminators predicted by TransTerm [26] are denoted as stem loop structures. Positions of the transcription start sites are indicated by arrows. B) The detailed sequences for the promoters $P_{\text {sugR }}, P_{p t s}, P_{c g 21 / 8}$, and $\mathrm{P}_{\text {pts }}$. Transcription starts $(+\mathrm{I})$, the distances to the translation start (in parentheses), and the start codons (underlined) are shown. The deduced- 10 and -35 promoter regions are highlighted by dark and light grey boxes, respectively. 


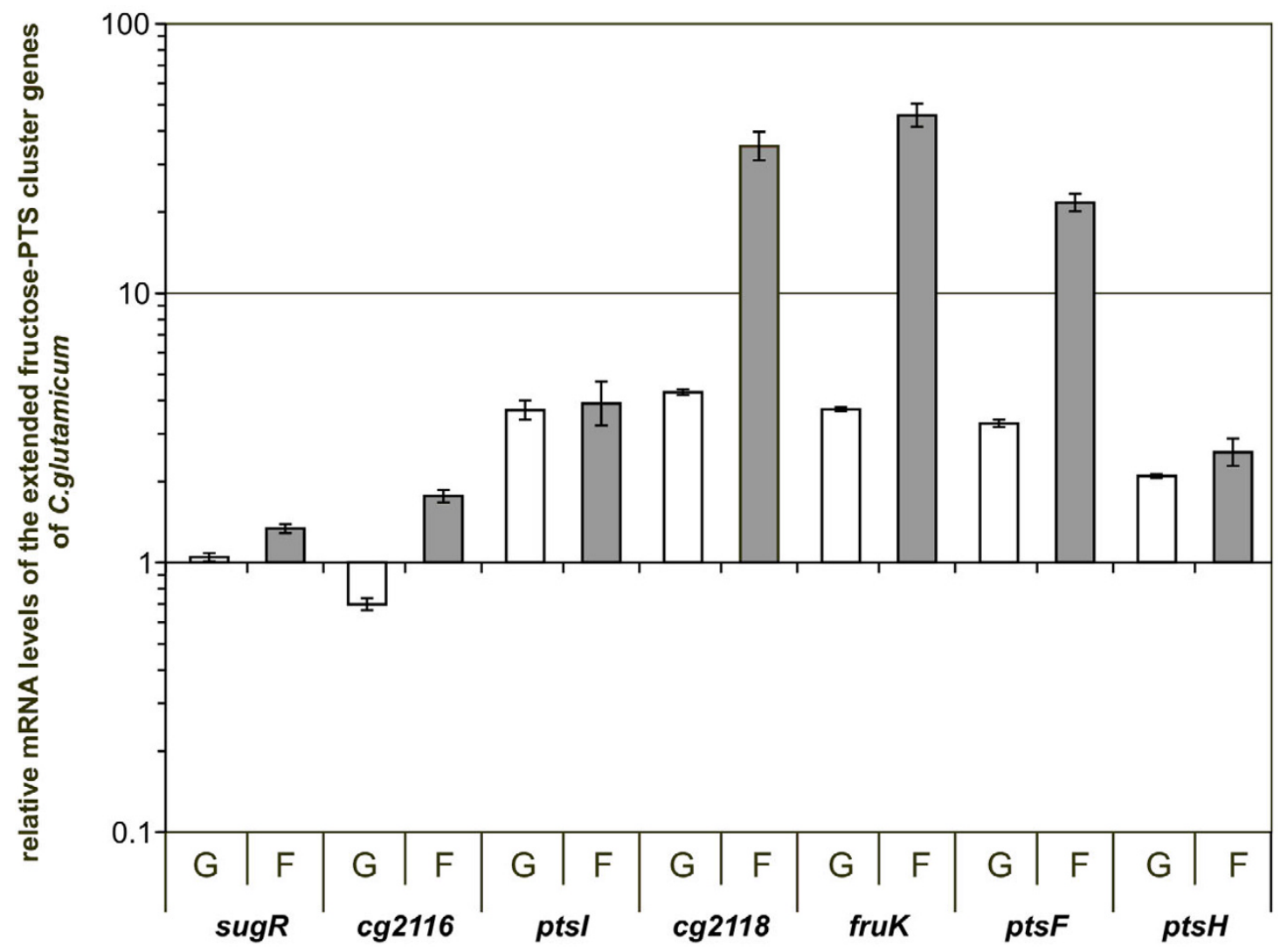

Figure 2

Transcriptional regulation of the extended PTS cluster genes of $C$. glutamicum cultures grown in liquid media containing glucose, fructose, or acetate. The strain RESI67 was grown in liquid media containing glucose, fructose, or acetate. By RT-PCR the mRNA levels of the genes sugR, cg2 I l6, ptsl, cg2 I I8, fruK, ptsF, and ptsH of cultures grown in glucose (white bars) or fructose (grey bars) were compared to those of cultures grown in acetate containing media. Results are means of four measurements from two biological replicates. Standard deviations are indicated by error bars.

tose-PTS gene cluster from $c g 2117$ (ptsI) to $c g 2120$ (ptsH) showed significantly higher mRNA levels during growth on glucose and fructose. The levels of the genes ptsI and ptsH did not differ between cells grown on glucose and fructose. This is in accordance with the need for expression in the presence of both sugars. In contrast to this, mRNA isolated from fructose grown cultures showed significantly higher transcription of $c$ 2118, fruK, and ptsF. The different expression patterns of $c g 2118$, fruK, and ptsF in cultures grown on glucose or fructose as sole carbon sources correlated with the requirement of the cell for a higher expression of the fructose-specific enzymeII ( $p t s F$ ) and the 1-phosphofructokinase encoded by fruK with fructose as the sole carbon source. These results are a clear indication for a carbon source-dependent transcriptional regulation of the genes of the PTS cluster.
The sugR gene is involved in the transcriptional regulation of the fructose-PTS cluster containing ptsl, cg2 I I 8, fruK, ptsF, and ptsH of C. glutamicum

Due to the classification of $s u g R$ and $c g 2118$ as DeoR-type regulators, they are both interesting candidate genes for investigating their involvement in the carbon sourcedependent transcriptional regulation of the fructose-PTS cluster genes. Single deletion mutants of $s u g R$ and $c g 2118$ were constructed by gene replacement in C. glutamicum RES167 in order to elucidate the possible roles in this regulation (data not shown). These deletions removed 242 of 780 nucleotides from the sugR coding region and 674 of 795 nucleotides from the $c g 2118$ coding region, rendering the respective gene non-functional and resulting in strains LG01 (RES167 $\Delta s u g R$ ) and LG02 (RES167 $\Delta c g 2118$ ), respectively.

The possible involvement of $s u g R$ or $c g 2118$ in transcriptional regulation of $p t s I$, the $c g 2118$-fruK-ptsF operon, and $p t s H$ was determined by real-time RT-PCR. For these 
experiments, total mRNAs isolated from RES167 and the mutant strains LG01 and LG02 were used. The cells were grown in shaking flasks in liquid minimal media with $2 \%$ acetate as sole carbon source. Acetate as the sole carbon source was chosen, because an increased transcription of the fructose-PTS cluster genes could be observed when RES167 was grown on glucose and fructose instead on acetate. Therefore, growth on acetate may cause the transcriptional repression of the fructose-PTS cluster genes. To find out whether the $s u g R$ or the $c g 2118$ gene product is involved in the transcriptional regulation of the fructosePTS cluster genes, the expression in the two deletion mutants LG01 and LG02 was compared to the expression in the RES167 strain when all strains were grown on acetate. The analysis revealed a strongly increased transcription of the fructose-PTS cluster genes ptsI, cg2118, fruK, $p t s F$, and $p t s H$ in the sugR mutant LG01 (Fig. 3). In contrast to this, the transcription of the fructose-PTS cluster genes in the cg2118 mutant LG02 was only weakly influ- enced. It is therefore concluded, that the sugR gene product downregulates transcription of the fructose-PTS gene cluster during growth on acetate, whereas cg2118 and its gene product does not seem to be involved in the transcription of the fructose-PTS cluster genes under the applied experimental conditions.

\section{The sugR gene of $C$. glutamicum is involved in the repression of PTS gene transcription}

Comparative microarray hybridization experiments with total mRNA isolated from the cg2115 deletion mutant LG01 and the parental strain RES167 both grown on acetate were conducted in order to explore the complete SugR regulon. In the microarray experiments using the $C$. glutamicum whole-genome DNA microarray described previously [28], two biological replicates were hybridized, simultaneously applying label-swapping. The results of the microarray experiments comparing the sugR mutant and the RES167 strain revealed 23 genes with increased

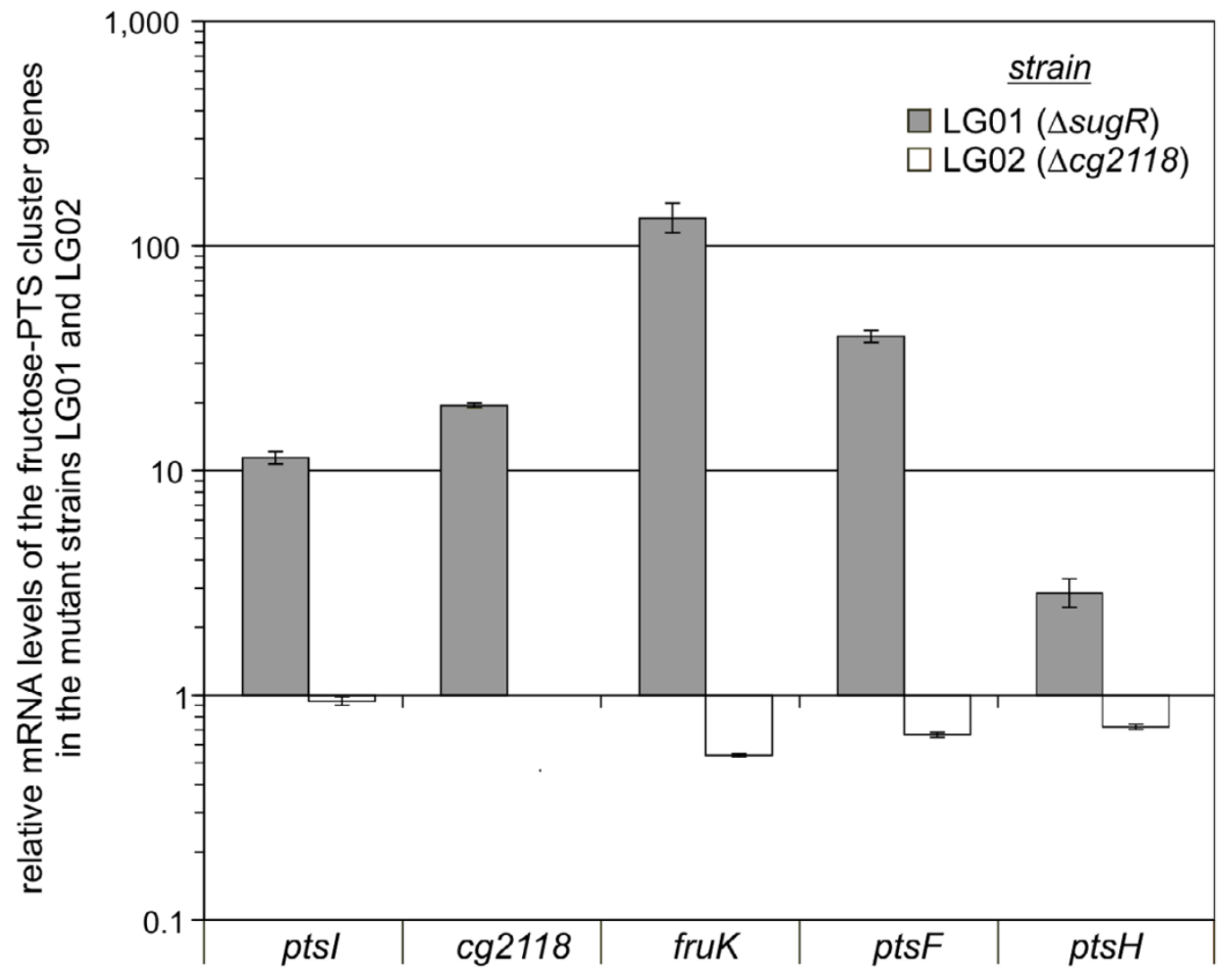

\section{Figure 3}

Relative gene expressions of $C$. glutamicum fructose-PTS cluster genes in dependence on the regulatory genes sugR and $c g 2$ I I 8 . The $C$. glutamicum strains LG0I, LG02, and RESI 67 were grown in liquid media containing acetate as sole carbon source. By RT-PCR the mRNA levels of the fructose-PTS cluster genes ptsl, cg2 I I8, fruK, ptsF, and ptsH of the mutant strains LG0I and LG02 were compared to those of RESI67. Due to the deletion of the cg2 I I 8 coding region, the expression of the truncated cg2 I / 8 gene in the mutant LG02 could not be determined. Results are means of four measurements from two biological replicates. Standard deviations are indicated by error bars. 
and five genes with decreased transcript levels in the sugR mutant. All genes of the fructose-PTS gene cluster were found in the group of genes with induced transcript levels (Fig. 4 and Table 2). Interestingly, the genes $p t s G$ and $p t s S$, which encode the enzyme II proteins for the uptake of glucose and sucrose, respectively, were also found to have an elevated expression level. To confirm the microarray results, subsequent real-time RT-PCR measurements were conducted, which validated the enhanced transcription of $p t s G$ and ptsS in the sugR mutant with ratios of 16.8 and 101.8 compared to RES167, respectively. Beside the PTS genes, the transcriptional levels of $l d h$ and $a d h A$ encoding lactate dehydrogenase and alcohol dehydrogenase, respectively, were found to be significantly increased. Significant increases in transcript levels were also observed for $c g 2025$ and $c g 2026$, encoding hypothetical proteins. Besides $l d h$ and $a d h A$, no further genes with known function in carbon metabolism were found to be significantly induced.
Since Engels and Wendisch [22] also compared global transcript levels by microarray hybridizations in a $\operatorname{sug} R$ mutant and its parent strain, the resulting stimulon can be compared to the one obtained in this work. In the intersection of the genes showing elevated transcript levels in both experiments, only the PTS genes mentioned above are apparent. The remaining genes corresponding to each stimulon are different between both studies and might be a result of the different growth conditions used (LB versus minimal media with acetate) or might be results of an indirect effect of the derepression of PTS gene transcription.

The SugR protein of C. glutamicum binds to sequences located upstream of the coding regions of ptsl, cg2 I | 8, ptsH, ptsG, and ptsS

To verify the direct involvement of the SugR protein in the regulation of the PTS gene cluster electrophoretic mobility shift assays (EMSA) were performed. For these experiments the SugR protein was purified after overexpression

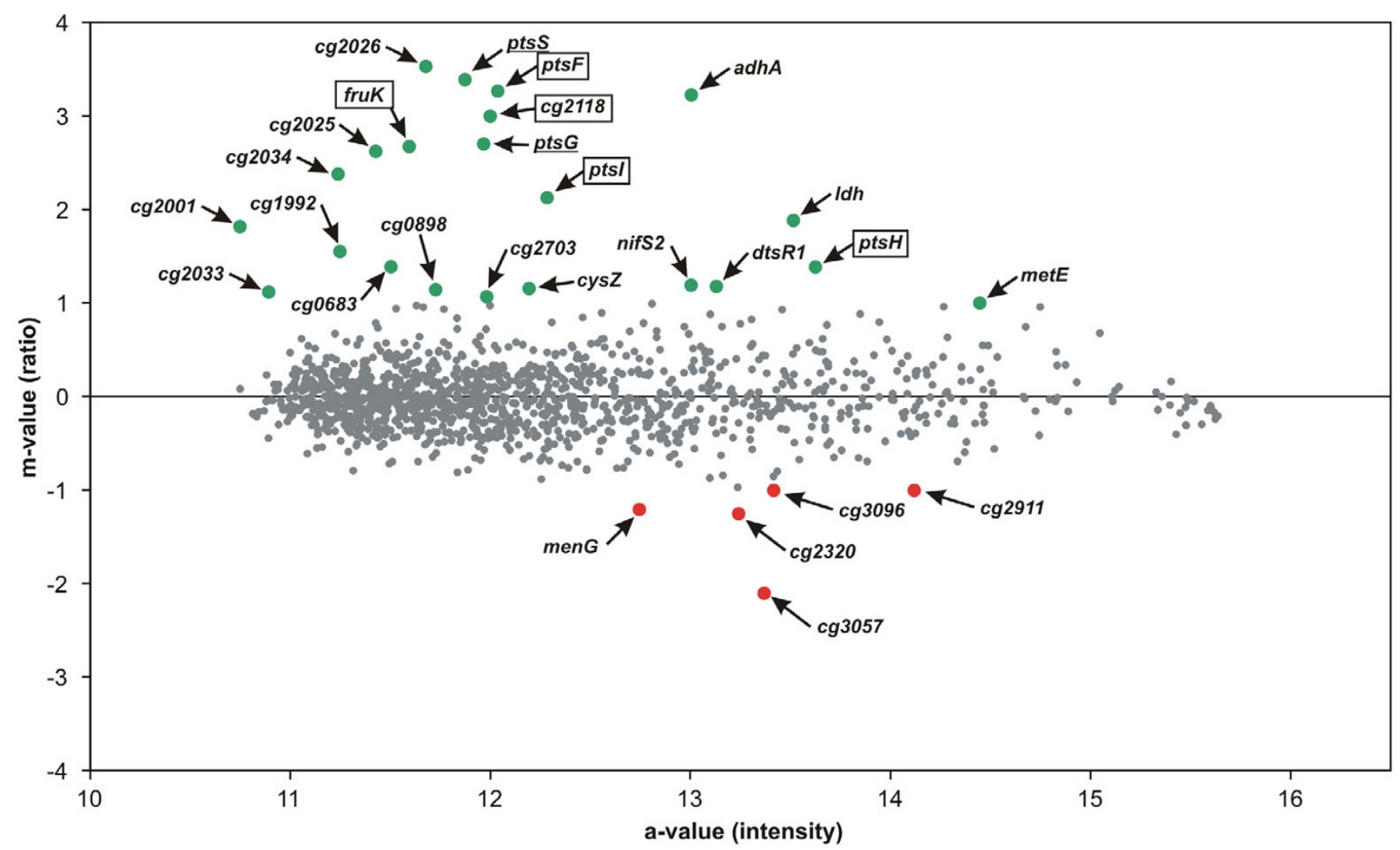

\section{Figure 4}

Analyses of the transcriptomes of the $C$. glutamicum sugR mutant LGOI and its parent strain RESI 67 by microarray hybridizations. The strains were grown in liquid minimal media containing acetate as sole carbon source. Global gene expression of LGOI was compared to that of RESI67 using microarray analyses performed on two biological and two technical replicates. Genes found to be significantly differentially expressed ( $m$-value $\geq \pm \mathrm{I}$ ) are indicated by green (elevated expression) or red (decreased expression) dots, respectively. Gene names belonging to the fructose-PTS gene cluster are boxed, and the non clustered PTS genes are underlined. 
Table 2: Differentially expressed genes deduced from the DNA microarray hybridization experiment comparing the transcriptomes of the sugR mutant LGOI with its parent strain RESI67 during growth on acetate

\begin{tabular}{|c|c|c|c|c|c|}
\hline CDS & NCBI No. & Gene name & m-value & a-value & Annotation \\
\hline cg2026 & - & - & 3.53 & 11.68 & Hypothetical protein \\
\hline cg2925 & $\mathrm{NCg} \mid 2553$ & pts $S$ & 3.39 & 11.87 & $\begin{array}{l}\text { Phosphotransferase system (PTS), sucrose-specific enzyme IIBCA } \\
\text { component }\end{array}$ \\
\hline $\operatorname{cg} 2120$ & NCgII86I & ptsF & 3.27 & 12.04 & $\begin{array}{l}\text { Phosphotransferase system (PTS), fructose-specific enzyme IIABC } \\
\text { component }\end{array}$ \\
\hline $\operatorname{cg} 3107$ & NCgl2709 & adhA & 3.23 & 13.00 & Alcohol dehydrogenase \\
\hline $\operatorname{cg} 2118$ & NCgII859 & - & 3.00 & 12.00 & Transcriptional regulator protein, DeoR-family \\
\hline $\operatorname{cg} 1537$ & $\mathrm{NCgII} 305$ & ptsG & 2.70 & 11.97 & $\begin{array}{l}\text { Phosphotransferase system (PTS), glucose-specific enzyme IIBCA } \\
\text { component }\end{array}$ \\
\hline $\operatorname{cg} 2119$ & $\mathrm{NCgl|} 860$ & fruk & 2.67 & 11.59 & I-Phosphofructokinase \\
\hline cg2025 & - & - & 2.62 & 11.43 & Hypothetical protein \\
\hline cg2034 & NCgl|739 & - & 2.38 & 11.24 & Hypothetical protein \\
\hline $\operatorname{cg} 2117$ & NCgII 858 & ptsl & 2.13 & 12.28 & Phosphotransferase system (PTS), Enzyme I \\
\hline $\operatorname{cg} 3219$ & NCgI28I0 & Idh & 1.88 & $|3.5|$ & L-Lactate dehydrogenase \\
\hline cg200I & NCgII 708 & - & 1.82 & 10.75 & Conserved hypothetical protein \\
\hline $\operatorname{cg} 1992$ & NCgII 699 & - & 1.55 & 11.25 & Hypothetical protein \\
\hline $\operatorname{cg} 2121$ & NCgII 862 & $\mathrm{pts} H$ & 1.39 & 13.62 & Phosphotransferase system (PTS), phosphocarrier protein $\mathrm{HPr}$ \\
\hline cg0683 & NCgI0565 & - & 1.39 & 11.50 & Putative permease \\
\hline cg2030 & NCgl|735 & - & 1.31 & 10.61 & Hypothetical protein \\
\hline $\operatorname{cg}|76|$ & NCgII 500 & nifS2 & 1.19 & 13.00 & Cysteine desulfhydrase \\
\hline $\operatorname{cg} 08 / 2$ & $\mathrm{NCgl0678}$ & $d t s R I$ & 1.18 & 13.13 & Acetyl/propionyl-CoA carboxylase, beta chain \\
\hline $\operatorname{cg} 3112$ & $\mathrm{NCg} \mid 27 \mathrm{I} 3$ & cys $Z$ & 1.15 & 12.19 & Sulfate transporter \\
\hline $\operatorname{cg} 0898$ & NCgl0754 & - & 1.14 & 11.73 & Pyridoxine biosynthesis enzyme \\
\hline cg2033 & NCgl| 738 & - & 1.12 & 10.89 & Putative secreted protein \\
\hline $\operatorname{cg} 2703$ & NCgl2373 & - & 1.07 & 11.98 & $A B C$-type putative sugar transporter, permease subunit \\
\hline $\operatorname{cg} / 290$ & $\mathrm{NCgII094}$ & metE & 1.00 & 14.45 & $\begin{array}{l}\text { 5-Methyltetrahydropteroyltriglutamate-homocysteine } \\
\text { methyltransferase }\end{array}$ \\
\hline $\operatorname{cg} 3096$ & $\mathrm{NCg} / 2698$ & - & -1.00 & 13.42 & Aldehyde dehydrogenase \\
\hline $\operatorname{cg} 2911$ & $\mathrm{NCg} / 2539$ & - & -1.00 & 14.12 & $A B C$-type putative $\mathrm{Mn} / \mathrm{Zn}$ transporter, substrate-binding lipoprotein \\
\hline $\operatorname{cg} 1055$ & NCgl0888 & menG & -1.21 & 12.74 & S-Adenosylmethionine:2-demethylmenaquinone methyltransferase \\
\hline $\operatorname{cg} 2320$ & NCgl2034 & - & -1.25 & 13.24 & Putative transcriptional regulator, ArsR-family \\
\hline $\operatorname{cg} 3057$ & NCgl2664 & - & -2.10 & 13.37 & Putative secreted protein \\
\hline
\end{tabular}

I Coding sequences (CDS) are numbered in reference to the complete genome sequence of $C$. glutamicum (GenBank accession number BX927/47) 2 Numbers in the genome database of the National Center for Biotechnology Information (NCBI No.)

in E. coli as an $\mathrm{N}$-terminal translational fusion with a selfcleavable intein tag using the pTYB1 vector [29]. The advantage of this system is that the native protein can be obtained without an additional attached tag sequence. The quality of the purified protein was controlled by 1dimensional SDS-PAGE and its size was estimated to be $\sim 28 \mathrm{kDa}$. This observation is in accordance with the annotation of the sugR coding region, which is predicted to encode a $27.6 \mathrm{kDa}$ protein with an N-terminal DeoRdomain comprising a helix-turn-helix $(\mathrm{HTH})$ motif. The identity of SugR was subsequently confirmed by peptide mass fingerprinting using MALDI-TOF mass spectrometry (data not shown).

As binding partners for SugR in the EMSA experiments, overlapping and fluorescently-labeled PCR fragments from the intergenic regions between ptsI and cg2118, as well as the regions upstream from the coding regions of sugR, ptsH, ptsG, ptsS, adhA, cg2025, and cg2026 were gen- erated. As negative controls, internal DNA fragments of the $c$ 2118- and $p t s H$-coding region were also produced by PCR and incubated with the purified protein to test for non-specific binding of the protein (data not shown).

For the gel retardation experiments $15 \mathrm{pmol}$ of the SugR protein and 0.05 pmol of the fluorescently-labeled PCR fragments were used. It was shown that SugR caused band-shifts in experiments with DNA fragments I1, I2, and I4 located in the ptsI-cg2118 intergenic region (Fig. 5). Furthermore, binding of the purified SugR protein was observed to the DNA fragment $\mathrm{H} 1$ which is located upstream of the $p t s H$ coding region, to the fragments G1 and G2 located upstream of the pts $G$ coding region and to the fragments S1 to S3 derived upstream of the coding region of $p t s S$.

The promoter region of $\operatorname{sugR}$ was also tested for SugR binding, but no shifts of the labeled PCR fragments were 


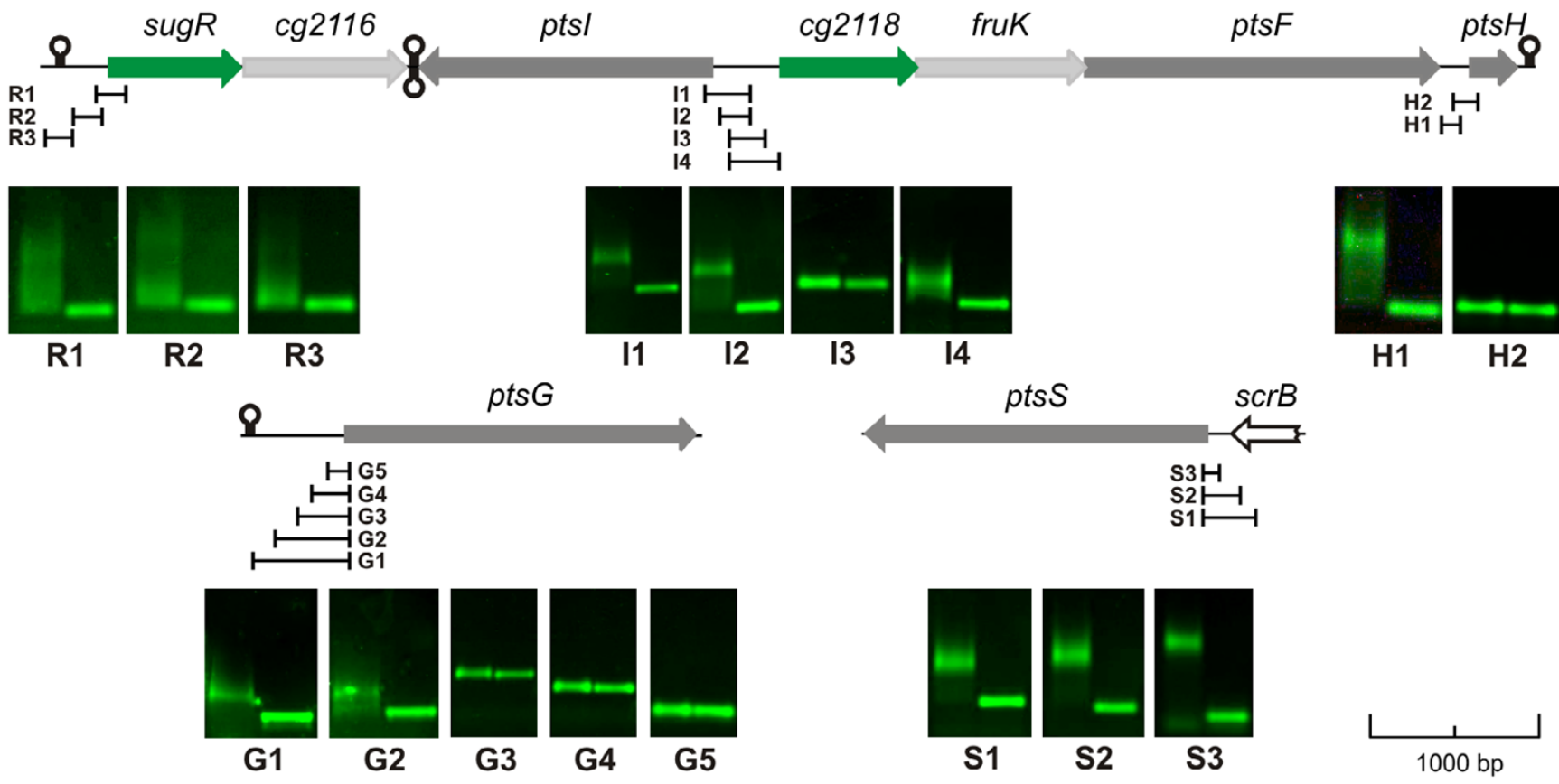

Figure 5

Electrophoretic mobility shift assays (EMSA) with selected upstream DNA fragments of PTS coding regions using the purified SugR protein. The physical maps of the extended fructose-PTS gene cluster as well as of the genes pts $G$ and ptsS are shown. Beneath the maps the fluorescently labelled PCR fragments are indicated which were used for EMSA studies. These studies were carried out with 15 pmol of purified SugR protein and 0.05 pmol of labeled PCR fragments. The results obtained for each PCR fragment are presented by agarose gel photos. In each picture, the left lane shows the shift in presence of the SugR protein, whereas the right lane shows the negative control without added SugR protein. Transcriptional terminators are denoted as stem loop structures.

detected (Fig. 5). Furthermore, labeled PCR fragments upstream of the $c g 2025, c g 2026$, and $a d h A$ coding regions were tested in EMSA experiments, but no binding of SugR was observed (data not shown).

The C. glutamicum SugR repressor binds to two 2 I bp DNA regions interfering with ptsl and cg2 I I 8-fruK-ptsF transcription

As described above, SugR binding was detected to the DNA fragments $\mathrm{I} 2$ and I4 located in the intergenic region between ptsI and cg2118. In contrast, no binding to the DNA fragment I3 was observed. These observations helped to identify two genetic regions A and B which are proposed to carry the specific binding sites for SugR (Fig. $6 \mathrm{~A})$. Region A is a $59 \mathrm{bp}$ long sub-region of the DNA fragment $\mathrm{I} 2$ and region $\mathrm{B}$ is a 87 bp long sub-region of I4. DNA sequence comparison of regionA to regionB revealed highly similar sequences of 21 nucleotides in length. To confirm these motifs as putative SugR binding sequences, double-stranded(ds)-oligonucleotides perfectly matching the 21 nucleotides with four flanking basepairs, representing the original genomic sequence on both sides, were used in competitive EMSA experiments and denoted as oligo A and oligoB, respectively (Fig. 6B). Interestingly, both oligonucleotides were able to displace the labeled DNA fragment I2. To further verify the binding sequence of SugR, ds-oligonucleotides with transversions of the four flanking basepairs of oligoA and oligoB, resulted in oligoAt and oligoBt, respectively, which were used in additional EMSA experiments (Fig. 6B). OligoAt and oligoBt, however, showed no differences in the competitory EMSA experiments compared to oligoA and oligoB, defining both $21 \mathrm{bp}$ long binding motifs as sufficient for SugR binding. According to the localization of the two SugR binding motifs in regionA and regionB of the DNA fragments $\mathrm{I} 2$ and $\mathrm{I} 4$, respectively (Fig. 6A), these two motifs were named motifA and motifB.

Subsequently, the positions of the two 21 bp SugR binding sequences motifA and motifB were mapped in the intergenic region between the coding regions of ptsI and cg2118 (Fig. 7). The two experimentally proven binding motifs in the intergenic region between ptsI and cg2118 are located within $\mathrm{P}_{c g 2118}$ and downstream of $\mathrm{P}_{p t s I}$ (motifA), or downstream the $\mathrm{P}_{c 82118}$ (motifB), respectively. Due to the divergent orientation of the coding regions $p t s I$ 
A)

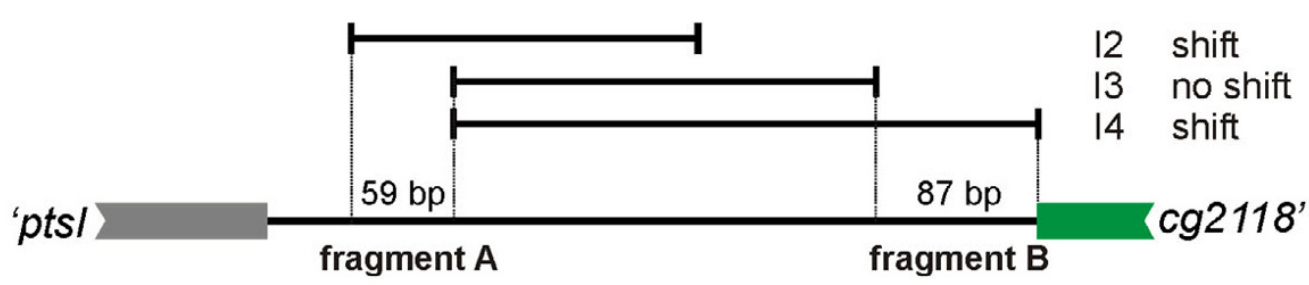

fragment A CAACAGTTGTGCAACTATTCAAACATTTGGATATTGACAAACAAACACATATCAACATA.

fragment $\mathbf{B}$ GGTTCT TCCACAATCGGAACCAAAACAACACTGGTCAAAAACCAGTTTCCCGCTTGAACAAAATTTCTTCCACGTCAACACCAACTC

B)

\author{
oligoA ATTGACAAACAAACACATATCAACATAGC \\ oligoB TTCCACAATCGGAACCAAAACAACACTGG
}
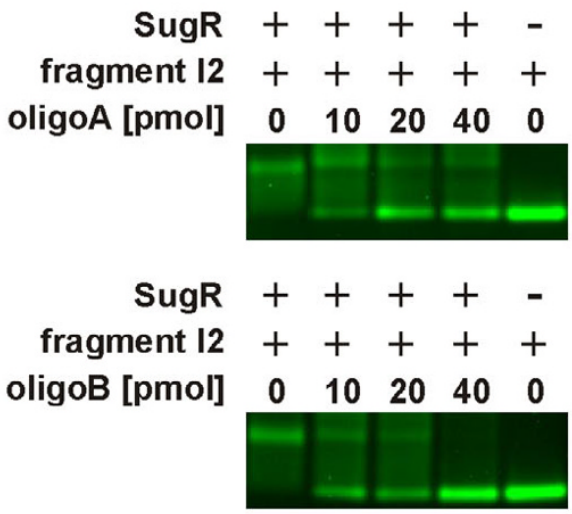

oligoAt CGGTACAAACAAACACATATCAACAGCTA

oligoBt GGAAACAATCGGAACCAAAACAACAAGTT

Figure 6

Identification of the SugR binding motifs by competitve EMSA studies. A) Detailed view of the pts//cg2 I / 8 intergenic region. For EMSA studies 15 pmol of the DNA fragments 12, 13, and 14 were used with 0.05 pmol of SugR protein. The DNA fragment I3 showed no binding of SugR, confining fragment $A$ and fragment B carrying one SugR binding motif each. Similar sequences representing putative binding motifs in the fragments $A$ and $B$ are highlighted in bold letters. $B$ ) Validation of the binding motifs by competitive EMSA experiments. OligoA and oligoB represent doublestranded(ds)-oligonucleotides carrying the similar sequences of fragment $A$ and $B$, respectively, with native bordering sequences. OligoAt and oligoBt represent those with modified bordering sequences. All four ds-oligonucleotides were used as competetive DNA fragments in EMSA experiments using 15 pmol purified SugR protein and 0.05 pmol of labeled DNA fragment 12. The 12/SugR-mixture was incubated with indicated amounts of the competitory (ds)-oligonucleotides. The mixture of the DNA fragment 12 and the SugR protein was used as positive control and the DNA fragment 12 alone as negative control.

and $c g 2118$ and the overlapping transcripts from the promoters $\mathrm{P}_{c g 2118}$ and $\mathrm{P}_{p t s I}$, binding of SugR to motifA will interfere with transcription from both promoters, whereas the binding of SugR to motifB will only interfere with the transcription from $\mathrm{P}_{c 82118}$.

To find additional binding sites of SugR, sequence alignments of motifA and motifB to the upstream coding regions of $p t s H, p t s G$ and $p t s S$ were conducted. In these alignments, only DNA regions which showed a band-shift in the EMSA experiments were used.

By this, two putative binding motifs were found upstream of the coding regions of $p t s H$ and $p t s G$ and $p t s S$ namely ptsH1, ptsH2 and ptsG1, ptsG2 and ptsS1, ptsS2 respectively (Fig. 8). Concluding, these experiments led to the identification of eight binding motifs. Two validated SugR binding sequences motifA and motifB located in the intergenic region of ptsI and $c g 2118$, as well as six putative 


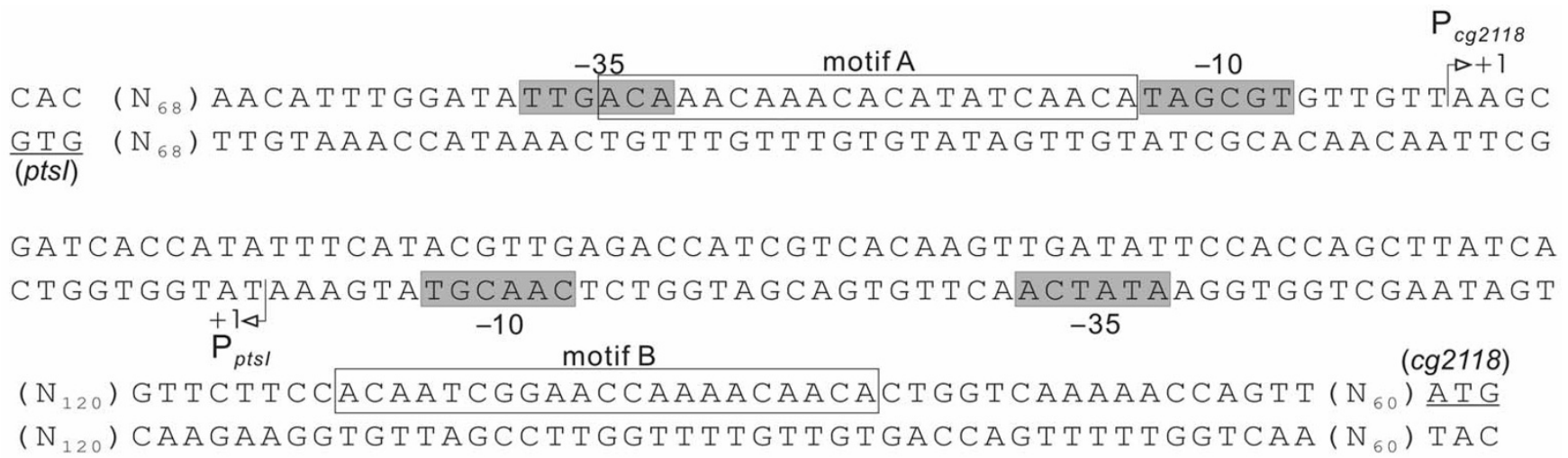

\section{Figure 7}

The organization of the intergenic region between the $C$. glutamicum genes pts/ and cg2 I I 8 containing the binding motifs $A$ and $B$. The double stranded sequence of the intergenic region between ptsl and cg2 I I 8 is shown. The experimentally proven binding motifs $A$ and $B$ are also boxed. The transcriptional start sites for the two genes are indicated as $P_{p t s l}$ and $P_{c g 2 / 18}$. The predicted -10 and -35 promoter regions are shown as dark grey boxes, respectively. The translational start codons of ptsl and cg2 I / 8 are underlined.

binding motifs located upstream of the coding regions of $p t s H$, ptsG, and ptsS. Sequence alignments of all eight motifs revealed that the six nucleotides ACAAAC in the 5'and the four nucleotides AACA in the 3'-region were well conserved (Fig. 8). Additionally, a predominant occurence was observed for cytosine and adenine nucleotides at positions 9 to 17 of the consensus motif. The characteristics of the putative consensus motif were determined as an $\mathrm{A}+\mathrm{C}$-rich and non-palindromic sequence (Fig. 8).

\section{The effectors fructose- I-phosphate, fructose- I,6- bisphosphate, and glucose-6-phosphate influence the binding activity of the SugR repressor}

In order to determine the effector substances influencing the activity of SugR, additional EMSA experiments were carried out. In these experiments, the SugR-I2 complex was incubated with fructose-1-phosphate (F-1-P) which exclusively occurs when fructose is taken up by the fructose-specific PTS. Additionally, glucose-6-phosphate (G6-P) occurring during the transport of glucose by the glucose-specific PTS component PtsG, fructose-1,6-bisphosphate (F-1,6-P) and fructose-6-phosphate (F-6-P), originating from the consumption of different sugars, as intermediates of glycolysis were used. Furthermore, the non-phosphorylated substrates of PtsF and PtsG, fructose and glucose, respectively, as well as acetate were used.

A loss of SugR binding to the DNA fragment I2 was observed to different extents using increasing concentrations of F-1-P, F-1,6-P, and G-6-P (Fig. 9). Using F-1-P as effector substance a clear effect at concentrations equal to and exceeding $10 \mu \mathrm{M}$ could be observed. In the case of F1,6-P, significantly higher concentrations of the effector substance ( $\geq 5 \mathrm{mM}$ ) for resolving the SugR-I2 complex had to be applied. Additionally, G-6-P showed significant influence on the SugR-I2 complex only at concentrations above $10 \mathrm{mM}$. The binding of SugR to the DNA fragment I2 was not influenced by F-6-P concentrations up to 60 $\mathrm{mM}$ (Fig. 9). Additionally, no influence was observed on SugR binding using the non-phosphorylated sugars glucose and fructose as well as acetate at concentrations up to $60 \mathrm{mM}$ (data not shown).

\section{Discussion}

The C. glutamicum regulatory gene sugR is involved in the repression of the PTS genes

In an initial study four putative transcripts were identified by RT-PCR covering $\operatorname{sug} R$ and $c g 2116$, which are co-transcribed, ptsI and $p t s H$, which are transcribed monocistronically, and the cg2118-fruK-ptsF operon. Coherent with this was the experimental identification of four promoters upstream of the coding regions of sugR, ptsI, cg2118, and ptsH.

In the C. glutamicum strain RES167 the transcript levels of $p t s I$, the $c g 2118$-fruK-ptsF operon and of $p t s H$ were considerably higher on the PTS sugars glucose and fructose in comparison to the non-PTS carbon source acetate. It is interesting to note, that the transcript levels of these five genes were moderately induced in glucose-grown cells, whereas in fructose-grown cells the transcript level of the cg2118-fruK-ptsF operon, specifically necessary for the transport and metabolism of fructose, was much stronger induced than that of the general PTS genes ptsI and $p t s H$. This indicates a differential activation of transcription for the general and the fructose-specific part of the fructosePTS gene cluster. 
A)

\begin{tabular}{cccc}
$\begin{array}{c}\text { motif } \\
\text { name }\end{array}$ & $\begin{array}{c}\text { upstream } \\
\text { of CDS }\end{array}$ & $\begin{array}{c}\text { proven binding sequences } \\
\text { predicted binding sequences }\end{array}$ & $\begin{array}{c}\text { distance } \\
\text { to TL }[\mathrm{bp}\end{array}$ \\
\hline A & ptsl /cg2118 & ACAAACAAACACATATCAACA & $\mathrm{N}_{84 / 323}$ \\
B & ptsl /cg2118 & ACAATCGGAACCAAAACAACA & $\mathrm{N}_{309 / 9}$ \\
ptsH1 & ptsH & ACAATGTCTCACTAGACTAAA & $\mathrm{N}_{126}$ \\
ptsH2 & ptsH & GGTAACGTGGCAAAACGAACA & $\mathrm{N}_{144}$ \\
ptsG1 & ptsG & ACATATTCAAAAGTATTACCT & $\mathrm{N}_{335}$ \\
ptsG2 & $p t s G$ & GCAGGTCGGACATATTCAAAA & $\mathrm{N}_{344}$ \\
ptsS1 & ptsS & ACAAACATTCATGTCTGAATA & $\mathrm{N}_{49}$ \\
ptsS2 & ptsS & TTGATCGGACACAAATAAACA & $\mathrm{N}_{96}$
\end{tabular}

B)

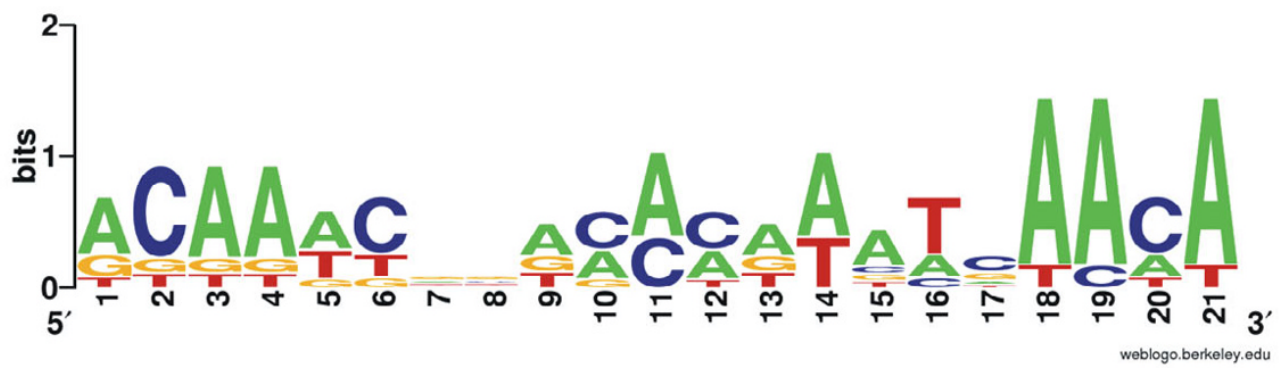

Figure 8

Comparison of experimentally determined and predicted SugR binding sites located upstream of the coding regions of ptsl, cg2 I l 8 , ptsH, ptsG, and pts and construction of a consensus sequence. A) Predicted binding motifs were determined by sequence comparisons of proven motifA and motifB to the DNA fragments showing positive SugR binding in the EMSA studies. Proven and predicted motifs are separated by a horizontal, dashed line. Boxed letters in the experimentally proven motifs $A$ and $B$ and the putative SugR binding sequences located upstream the coding regions of pts $H$, pts $G$, and pts $S$ denote identical nucleotides in all sequences. Distances to the according translation starts (TL) are indicated. B) A frequency plot of the deduced consensus sequence of all motifs is constructed by means of the WebLogo tool. The overall height of each stack of letters indicates the sequence conservation at each position of the 21 -bp motif, whereas the height of each symbol within the stack reflects the relative frequency of the corresponding nucleotide at that position.

Microarray hybridizations comparing global transcript levels in the C. glutamicum sugR mutant LG01 and its parent strain, both grown on acetate, helped to confine the genes belonging to the stimulon of the LG01 mutant. For this, it was interesting to note that together with the genes of the fructose-PTS cluster, the distantly located PTS genes $p t s G$ and $p t s S$ were also found to be derepressed in the sugR mutant. These results were subsequently verified by using the more sensitive real-time RT-PCR technique verifying the expansion of the putative SugR regulon to the non-clustered PTS genes $p t s G$ and $p t s S$. 
A)

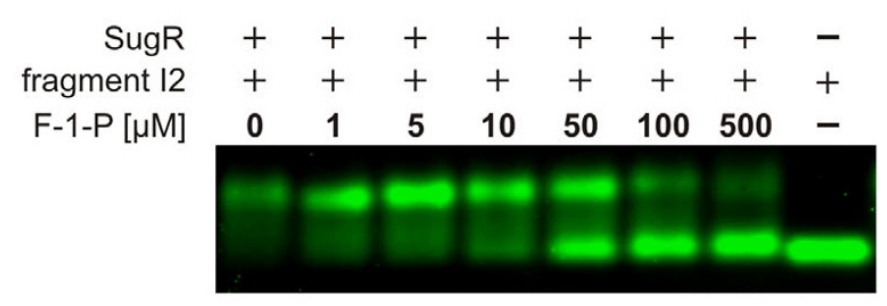

C)

$\begin{array}{rccccccc}\text { SugR } & + & + & + & + & + & + & - \\ \text { fragment I2 } & + & + & + & + & + & + & + \\ \text { F-6-P [mM] } & 0 & 1 & 3 & 10 & 30 & 60 & - \\ & & & & & & & \end{array}$

B)

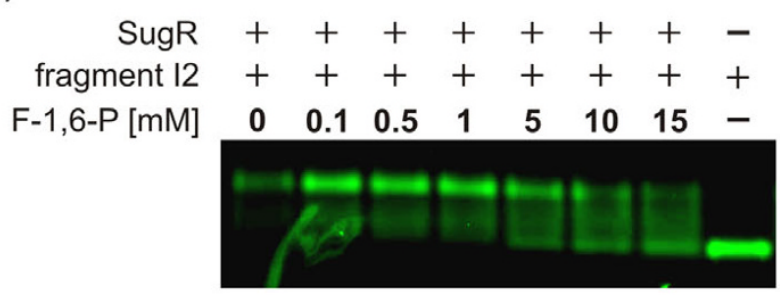

D)

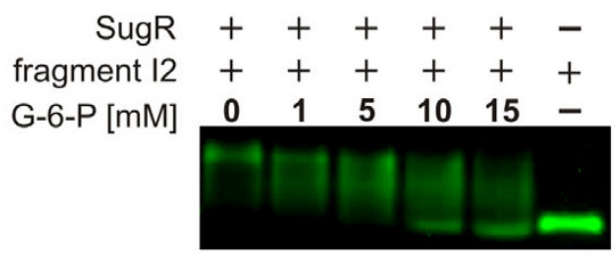

\section{Figure 9}

Identification of effector substances inactivating the repressor protein SugR. Electrophoretic mobility shift analysis of I5 pmol purified SugR protein complexed with 0.05 pmol of the DNA fragment 12 in the presence of different putative effectors were conducted. Effectors applied to the SugR/I2-complex at varying concentrations as described were A) fructose-I-phosphate (FI-P), B) fructose-I,6-bisphosphate (F-I,6-P), C) fructose-6-phosphate (F-6-P), and D) glucose-6-phosphate (G-6-P)

In a parallel study, Engels and Wendisch [22] analyzed the same regulatory genes by constructing mutants and analysing effects on the regulation of PTS genes. They initially focussed on transcriptional regulation of $p t s G$ and showed that this gene was transcriptionally regulated by the product of the sugR gene. By using microarray analysis to compare the global transcription levels of the sugR mutant to the parental strain grown on LB medium, they also ensured that $p t s S$ and the genes $c g 2118$ and $p t s F$ are under the same transcriptional control. However, in their study the gene carrying the regulatory function was named sugR. By our microarray results, we were able to extend the SugR regulon by adding fruK and the genes encoding the general part of the PTS, ptsI and ptsH. Hence, this work shows that all functionally characterized PTS genes are under the control of the SugR regulator.

In our microarray analysis, differential regulation of the genes cg3365 and cg3366, putatively encoding a PTS component of a hitherto unknown function, was not detected. Therefore, these genes might not be a part of the SugR regulon. In contrast to this, elevated transcript levels were detected for the genes cg2025 and cg2026, encoding proteins of unknown function, and $a d h A$, putatively encoding a Zn-dependent alcohol dehydrogenase. But, EMSA studies only confirmed the in vitro binding of SugR upstream to the according coding regions of the PTS genes. The significant expression changes of $c g 2025, c g 2026$, and $a d h A$ are therefore most likely indirect effects of the sugR mutation.

An autoregulation of SugR is unlikely, because real-time RT-PCR experiments showed only marginal deviations in the transcriptional regulation of the sugR-cg2116 operon in the $\operatorname{sugR}$ mutant in comparison with its parent strain. Coherent with this, binding of purified SugR protein upstream of the coding sequence of $s u g R$ was not detected.

\section{The DeoR-type regulator SugR acts as a repressor on transcription of all PTS genes in C. glutamicum}

In electrophoretic mobility shift assays (EMSA), binding of the native, purified SugR protein to DNA fragments located upstream of the coding sequences of ptsI, pts $H$, $p t s G$, ptsS, and the cg2118-fruK-ptsF operon was detected. This result substantiated the identification of these genes as part of the SugR regulon. Binding of SugR to its target genes occurs in the absence of effectors and transcription is negatively affected as described above. Therefore, SugR acts as repressor of transcription on PTS genes.

The results obtained in this work correlate well with the current knowledge, where DeoR-type regulators of Grampositive bacteria often act as repressor of sugar-specific PTS genes responsible for the uptake and metabolism of sugars like fructose (Lactococcuslactis, [13]; Streptococcus gordonii, [15]), lactose (L. lactis, [30]; Staphylococcus aureus, [31]), and sorbose (L. casei; [32]). In Bacillus subtilis DeoR- 
type regulators are also involved in the transcriptional regulation of deoxyribonucleoside degradation [33]. The function of DeoR-type regulators was further investigated in the Gram-negative organisms E. coli and Salmonella enterica where they have transcriptional influences on non-PTS operons involved in the metabolism of deoxyribose [34,35]. A common feature of the described DeoRtype regulators is the transcriptional control restricted to neighboring genes. Solely the DeoR-type regulator DeoT of $E$. coli has potential target genes of various metabolic pathways distributed in diverse genetic loci and was designated as a master regulator [36]. In accordance to the distribution of the PTS genes in the C. glutamicum genome, the SugR repressor can be designated as a pleitropic regulator of the PTS genes.

\section{The SugR binding site comprises a 2 I-base pair AC-rich motif}

The sequence comparisons of the SugR binding motifs led to a putative consensus sequence, which consists of two conserved non-palindromic flanking regions and an ACrich center region. The DeoR regulators described for $E$. coli (DeoR), B. subtilis (DeoR), and Salmonella enterica (DeoQ) [34,37,35] are recognizing palindromic sequences, as well as the the DeoR-type regulator which is responsible for the transcriptional regulation of the fructose-pts gene cluster in Streptococcus gordonii (FruR) [15]. In addition the DeoR-type regulator of the fructose-PTS gene cluster of Lactococcus lactis (FruR) potentially binds to four repeating non-palindromic sequences [13]. The putative consensus sequence of the SugR binding site, however, neither shows significant similarity to the known palindromic binding sequences, nor to the nonpalindromic sequences of DeoR-type repressors mentioned above.

In their parallel study, Engels and Wendisch [22] studied the binding of SugR (SugR), modified and purified by an aminoterminal decahistidine tag, to the $p t s G$ upstream region. By comparing the results obtained from EMSA experiments using overlapping DNA fragments, they confined the binding site to a 23 bp sequence in which they found an 8 nt motif (5'-GTCGGACA-3') which is partly conserved in the upstream regions of ptsS and $c g 2118$. Although the possibility remains that the SugR binding site is shorter than the 21 nucleotides as predicted here, the 8 nt possibly involved in the binding of SugR as defined by Engels and Wendisch [22] is included in a putative $21 \mathrm{nt}$ sequence (ptsG2) postulated in this work.

The two experimentally determined SugR binding motifs A and B are located in the intergenic region between ptsI and $c g 2118$ and are therefore of special interest. On one hand, motifA is overlapping the promoter region of $\mathrm{P}_{c g 2118}$ and located downstream of $\mathrm{P}_{p t s I^{\prime}}$ on the other hand
motifB is located downstream the promoter $\mathrm{P}_{c g 2118}$. The binding within or downstream the promoter region is common to transcriptional repressors [38]. Therefore, the binding of SugR in the operator region of $p t s I$ and $c g 2118$ apparently results in the repression of the transcription of the divergently orientated genes. Even though the SugR binding sites upstream of the pts $G$ coding region, detected in the work of Engels et al. [22] and this work are located upstream the promoter $P_{p t s G}$ a spacing of $30 \mathrm{bp}$ to the $35^{\text {th }}$ nucleotide may be sufficient for transcriptional repression by SugR. However, the maximal repression of the deo operon in E. coli is produced by DeoR binding to three operator sequences which are overlapping and located upstream of the transcription start sites [34], resulting in cooperative binding of different regulatory sequences leading to gene repression [39]. Such behaviour is putatively not present in C. glutamicum since a SugR binding to DNA fragments located between transcription and translation start (fragments G3 to G5; Fig. 5) was not observed. However, further binding sites located in the coding region of $p t s G$ were not ruled out by experimental approaches and therefore the repression of $p t s G$ transcription by SugR remains unclear.

\section{Fructose-I-phosphate and other sugar phosphates act as effectors releasing SugR from its binding sites}

The strong effect of F-1-P on SugR binding to the ptsI$c g 2118$ intergenic region is very interesting, since F-1-P is a metabolite mainly occuring during the uptake of fructose mediated by PtsF. Therefore, F-1-P is an ideal intracellular substance for sensing the presence of external fructose and efficiently induces expression of the genes encoding the general and fructose-specific parts of the PTS, $p t s I, p t s H$, and $p t s F$, respectively. Once external fructose is consumed, the F-1-P concentration is reduced by the activity of 1-phosphofructokinase encoded by fruK converting F-1-P to the less effective metabolite F-1,6-P. Although, the internal concentration of F-1-P in $C$. glutamicum grown on glucose or fructose is unknown, the internal concentrations of the effector substances G-6-P could be determined to $8 \mathrm{mM}$ or $15 \mathrm{mM}$ and of F-1,6-P to $23 \mathrm{mM}$ or $46 \mathrm{mM}$ grown on glucose or fructose, respectively. Comparing the internal amounts of G-6-P and F1,6-P to the amounts used for the EMSA-studies suggests that SugR is negatively influenced by theses effectors in vivo. During the consumption of the F-1,6-P pool, the regulatory system might return to a maximal repressed state. The effector substance F-1-P was also shown to specifically affect the global regulator Cra of E. coli $[40,41]$. It is furthermore described as the main effector for the FruR repressors of the fructose operon in Streptococcus gordonii [15] and Spiroplasma citri [14].

Besides the intermediate occuring during the consumption of glucose and fructose, namely F-1,6-P, the product 
of glucose uptake via the PTS, namely G-6-P, is apparently able to affect SugR binding activity and to derepress transcription of the PTS genes. This would be necessary at least for the genes of the general part of the PTS (ptsI, ptsH) and for the distantly located $p t s G$ gene. However, much higher concentrations of F-1,6-P or G-6-P are necessary for resolving the DNA-SugR protein complex. These results were confirmed by the RT-PCR data for the wildtype, where the transcription of the fructose-PTS cluster genes of cells grown on glucose did not reach the induction levels of cells grown on fructose, indicating higher activity of SugR on glucose then on fructose and resulting in higher induction levels of the fructose-PTS cluster genes on fructose.

As a striking difference, Engels and Wendisch [22] identified F-6-P as the only effector of SugR (SugR) when bound to the $p t s G$ upstream region. F-6-P is the successive metabolic intermediate produced from G-6-P by the phosphoglucose isomerase. Therefore, F-6-P is a suitable candidate for the cell to determine whether glucose is externally available.

In the experiments performed with the ptsI-cg2118 intergenic region, no effect on SugR binding was observed for F-6-P up to concentrations exceeding $20 \mathrm{mM}$, which were applied for full resolution of the complex between SugR (SugR) and the ptsG upstream region in the study of Engels and Wendisch [22]. However, internal concentrations of $13 \mathrm{mM}$ or $3 \mathrm{mM}$ and $1 \mathrm{mM}$ or $2 \mathrm{mM}$ F-6-P were determined for C. glutamicum grown on glucose and fructose, respectively $[42,19]$, ranging below the amounts used for the EMSA studies here.

In addition, the divergent promoters $\mathrm{P}_{p t s I}$ and $\mathrm{P}_{c g 2118}$ are coordinately regulated as shown by the mRNA measurements in the sugR mutant compared to the parental strain. However, divergently transcribed genes were already described, e.g. for the C. glutamicum genes aceA and aceB [43]. The corresponding promoters partly overlap by their -10 region and regulation of the divergent genes ace $A$ and $a c e B$ is obtained by binding of the regulator RamB to one binding site located in the intergenic region [44]. Hence, switching between the repressive and the activated state is coordinately triggered. Nevertheless, the aceA and aceB promoters are only overlapping by their promoter sequence. The promoters $\mathrm{P}_{p t s I}$ and $\mathrm{P}_{c g 2118}$ are producing transcripts, which are complementary by 14 nucleotides, whereby the overlapping mRNA transcripts do not influence the regulatory coupling of the divergent genes ptsI and $c g 2118$, but represent a hence unique promotor structure in C. glutamicum.

As mentioned above, binding of SugR to motifB only interferes with $c g 2118$-fruK-ptsF transcription providing the opportunity for the cell to differentially regulate expression of the fructose-specific part, and the ptsI and $p t s H$ gene of the general part of the PTS as observed for fructose and glucose grown cells. In detail, the expression of the PTS gene cluster in C. glutamicum grown on acetate was compared to the one on fructose and glucose, respectively, indicates that the complete gene cluster is derepressed in the presence of glucose. In the presence of fructose, however, the expression of the fructose-specific genes fruK and $p t s F$ is significantly higher than the expression of $p t s I$ and $p t s H$. Therefore, the level of the transcription from $\mathrm{P}_{c g 2118}$ is somehow further adjusted by the actual carbon source, whereas the transcription from $\mathrm{P}_{\text {pts }}$ and $\mathrm{P}_{p t s H}$ remains unaffected. At the moment it is not clear how this differential regulation, affecting the transcription from $\mathrm{P}_{c 22118^{\prime}}$ is exerted. It is possible that the SugR-DNA complexes react differentially to different effectors as it is shown for the SugR (SugR) binding site upstream of the coding region of $p t s G$ [22]. This differential behaviour might be interpreted in a sense that SugR is able to form complexes with individual binding sites that have different sensitivities to sugar phosphates. A sugar specific regulation of different genes was also observed in the hyperthermophilic archaeon Pyrococcus furiosus, where the transcriptional regulator of the trehalose/maltose and the maltodextrin $\mathrm{ABC}$ transporter $\operatorname{TrmB}$ is inactivated by the effectors maltose and trehalose or maltotriose and sucrose, respectively $[23,24]$. However, the regulation of different genes by recognition of varying effectors could be an adequate explanation for the differing effectors determined by Engels [22] and this work. Furthermore, it can not be excluded that other regulators may play a role in the differential expression of the PTS gene cluster. The function of the second DeoR-type regulator gene located in the PTS gene cluster cg2118 in transcriptional regulation of the PTS gene cluster remained unclear. The deletion mutant of cg2118, however, showed no influence on transcription of the PTS genes under the tested conditions, therefore leaving the potential targets of Cg2118 unknown. However, a sugR/cg2118 double mutant (strain LG03) was constructed and investigated with RT-PCR compared to the $\operatorname{sug} R$ single mutant, but no further induction of the transcription levels were observed for the fructose PTS cluster genes when both strains were grown on fructose or acetate (data not shown). Furthermore, the Cg2118 protein was purified by the IMPACT-method described and EMSA-studies were carried out with the intergenic region of $p t s I / c g 2118$ and the upstream region of ptsH. Binding of Cg2118 to the corresponding DNAfragments was not detected (data not shown). Further microarray experiments with the deletion mutant of cg2118 will be a subject of further studies and may reveal the function and the regulon of $\mathrm{Cg} 2118$. 


\section{Conclusion}

The detailed investigation of the transcriptional regulation revealed the DeoR-type regulator SugR to be responsible for the repression of the fructose-PTS cluster genes $p t s I, c g 2118$, fruK, ptsF, and $p t s H$, as well as of the distantly located PTS-genes $p t s G$ and $p t s S$. These results were confirmed by the determined promotor and SugR-binding sequences, extending the knowledge on PTS-dependent transcriptional regulation.

The main negative effector of SugR with regard to the fructose-PTS genes (F-1-P), was different to that identified in the study concentrating on the regulation of ptsG (F-6-P) by Engels and Wendisch [22]. In combination, these results indicated that SugR is able to regulate genes in dependence on the presence of different effectors, reflecting a hitherto unknown regulatory mechanism in Corynebacteria.

It remains to be assessed whether the arrangement and sequence composition of the SugR-binding sites are responsible for the recognition of different effectors by SugR. Further experiments dealing with the mutational analyses of the SugR-binding sites could clarify this issue.

\section{Methods}

\section{Strains and media}

Bacterial strains and plasmids used in this study are listed in Table 3. All C. glutamicum strains were cultivated in shaking flasks at $30^{\circ} \mathrm{C}$ with $150 \mathrm{rpm}$ in minimal medium MM1. The growth medium contained $2 \%(\mathrm{w} / \mathrm{v})$ carbon source dependent on the test setup (glucose, fructose, and sodium-acetate), $\quad 1 \% \quad\left(\mathrm{NH}_{4}\right)_{2} \mathrm{SO}_{4}, \quad 0.3 \%$ urea, $0.1 \%$ $\mathrm{K}_{2} \mathrm{HPO}_{4} ; \mathrm{pH}$ 7.2. After autoclaving the following substrates were added: $50 \mu \mathrm{gl}^{-1}$ biotin and $0.1 \%(\mathrm{v} / \mathrm{v})$ trace element solution containing $2 \mathrm{gl}^{-1} \mathrm{FeSO}_{4} \times 7 \mathrm{H}_{2} \mathrm{O}, 2 \mathrm{gl}^{-1}$ $\mathrm{MnSO}_{4} \times 1 \mathrm{H}_{2} \mathrm{O}$ and $50 \mathrm{gl}^{-1} \mathrm{NaCl}$. E. coli DH5 $\alpha \mathrm{MCR}$ and JM109 were grown in shaking flasks at $37^{\circ} \mathrm{C}$ with 150 rpm in Luria-Bertani (LB) medium [45]. For plasmid selection the following antibiotics were used: ampicillin (100 $\mu \mathrm{gml}^{-1}$ for E. coli), kanamycin $\left(50 \mu \mathrm{gml}^{-1}\right.$ for E. coli and $25 \mu \mathrm{gml}^{-1}$ for C. glutamicum), and nalidixic acid 50 $\mu \mathrm{gml}^{-1}$ for C. glutamicum selection.

\section{PCR techniques}

PCR experiments were performed by using the DNA Engine Dyad thermocycler (PTC-0220) from MJ research Inc. (Watertown, Mass.). Oligonuleotides were obtained from Operon (Qiagen, Germany). Amplification of DNA was performed with Phusion Hot Start DNA polymerase (New England Biolabs, USA), which features proof-reading activity. Initial denaturation of the template DNA was carried out at $96^{\circ} \mathrm{C}$ for $4 \mathrm{~min}$. The PCR cycle started with denaturation for $30 \mathrm{~s}$, followed by annealing at primer dependent temperature at $\mathrm{T}_{\mathrm{m}}(2 \mathrm{AT}+4 \mathrm{GC})[46]$, and extension at $72^{\circ} \mathrm{C}$ whereas amplification time was chosen corresponding to fragment length and speed of the polymerase. The cycle was repeated 34 times, followed by a final extension step for $7 \mathrm{~min}$ at $72^{\circ} \mathrm{C}$. Purification of the PCR products was carried out by using a PCR Purification Spin kit (Qiagen, Hilden, Germany).

\section{DNA isolation, transfer and manipulation}

E. coli DH5 $\alpha$ MCR was used for routine recombinant DNA experiments. Plasmid DNA of E. coli was isolated by means of the GenElute bacterial DNA isolation kit (Sigma Aldrich, Steinheim Germany). DNA-modification, analysis by gel-electrophoresis, and ligation were applied as standard procedures [45]. Restriction endonucleases and T4 DNA ligase were obtained from Amersham-Pharmacia (Freiburg, Germany) and Roche (Mannheim, Germany). Plasmid DNA was introduced into E. coli and C. glutamicum strains by electroporation as described previously $[47,48]$ employing the Bio-Rad Gene Pulser system (BioRad, Munich, Germany).

\section{Construction of the C. glutamicum deletion mutant strains}

The plasmids pLMJ1 and pLMJ2 carrying deletion fragments of the genes $\operatorname{sugR}$ and $c g 2118$ were constructed using the GeneSOEing method based on the PCR-mediated recombination as described by Horton et al. [49] (Table 3). Artificial MunI- and BamHI-sites for sugR and BglII- and EcoRI-sites for cg2118 were added by 5'-primer extension on both ends of the deletion fragment. Subsequently the resulting fragments were cleaved by the corresponding restriction enzymes and cloned into the pK18mobsacB vector. The resulting plasmids pLMJ1, pLMJ2 were transferred by electroporation into C. glutamicum RES167 [47] and therefore used to introduce the deletions by homologous recombination into C. glutamicum [50]. Thus the C. glutamicum mutant strains LG01, LG02, and LG03 carrying deletions of the genes sugR, cg2118, and a $s u g R / \operatorname{cg} 2118$ double deletion were obtained.

The sugR, cg2118, and sugR/cg2118 deletion mutant strains were subsequently verified by PCR using additional primers positioned outside of the deletion construct as described by Rückert et al. [51]).

\section{Genetic construction, expression and purification of heterologous expressed Intein-coupled protein SugR}

The SugR protein was purified by a translational fusion to intein and subsequent affinity purification performed by the IMPACT-CN system (New England Biolabs, USA), which allows the purification of the target protein without any remaining tag by thiol-induced self-cleavage of the intein. The sugR expression plasmids (pLGI1) was constructed by PCR amplification of a 777 bp fragment, including the complete sugR gene, except the stopcodon 
Table 3: Bacterial strains and plasmids used

\begin{tabular}{|c|c|c|}
\hline Strain or plasmid & Relevant markers, phenotypes, and characteristics & Reference or origin \\
\hline \multicolumn{3}{|l|}{ C. glutamicum strains } \\
\hline RESI67 & 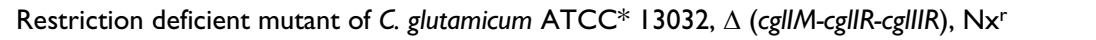 & [47] \\
\hline LG0I & RESI67 with sugR deletion, after double crossover with pLMJI, Nx ${ }^{r}$ & This work \\
\hline LG02 & RESI 67 with cg2 I / 8 deletion, after double crossover with pLMJ2, Nxr & This work \\
\hline LG03 & $\begin{array}{l}\text { RESI67 with cg2 I /8/sugR double-deletion, after double crossover with pLMJI and pLMJ2, } \\
N x^{r}\end{array}$ & This work \\
\hline \multicolumn{3}{|c|}{${ }^{\prime}$} \\
\hline ER2566 & $\begin{array}{l}\text { F-ë-fhuA2 [lon] ompT lacZ::T7 gene I gal sulA|I } \Delta(\text { mcrC-mrr) I I4::ISIO R(mcr-73::miniTn I0- } \\
\text { TetS)2 R(zgb-2 I0::Tn I0)(TetS) endAI [dcm] }\end{array}$ & New England Biolabs \\
\hline JMI09 & $\begin{array}{l}\text { recAI, endAI, gyrA96, thi, hsdRI7, supE44, relAI, } \Delta(\text { lac-proAB)/F' [traD36, proAB', lacla, } \\
\text { lacZ } \triangle M I 5]\end{array}$ & Takara Bio Inc. \\
\hline LG2I & JMI09 with expression vector pLGII for plasmid isolation, $A^{r}$ & This work \\
\hline LG3I & ER2566 with expression vector pLGII for the overexpression of SugR, Apr & This work \\
\hline \multicolumn{3}{|c|}{ - } \\
\hline pKI8mobsacB & $\begin{array}{l}\text { mobilizable E. coli cloning vector, allows for double crossover in C. glutamicum, sacB, lacZ } \alpha \text {, } \\
\mathrm{Km}^{r}\end{array}$ & {$[50]$} \\
\hline pZErO-2 & E. coli vector, lac promoter, lacZ $\alpha, \operatorname{ccdB}$ lethal gene, $\mathrm{Km}^{\mathrm{r}}$ & Invitrogen \\
\hline PTYBI & E. coli expression vector, C-terminal intein tag, $\mathrm{T} 7$ promoter, lacl, rrnB TI, Ap ${ }^{r}$ & New England Biolabs \\
\hline pLMJI & $\begin{array}{l}\text { pKI } 8 \text { mobsacB containing } 588 \text { bp sugR deletion fragment (sugR-d I/4), obtained by EcoRI- } \\
\text { BamHI fusion, } \mathrm{Km}^{r}\end{array}$ & This work \\
\hline pLMJ2 & $\begin{array}{l}\text { pK I 8mobsacB containing III7 bp cg2 I I } 8 \text { deletion fragment (cg2II8-dI/4), obtained by Bglll- } \\
\text { EcoRI fusion, } \mathrm{Km}^{r}\end{array}$ & This work \\
\hline pLGII & PTYBI containing sugR (777 bp), obtained by Ndel-Sapl fusion, Apr & This work \\
\hline
\end{tabular}

* American Type Culture Collection

to verify C-terminal fusion of the desired protein with a 55 $\mathrm{kDa}$ intein-tag of the pTYB-1 vector (New England Biolabs, USA). At the $5^{\prime}$ end an artificial NdeI-site and at the 3' end of the fragment an artificial SapI-site was added by $5^{\prime}$ primer extension. As recommended by the vendor the NdeI-site contains an ATG sequence for translation initiation and the SapI-site places the C-terminus of the target protein immediately adjacent to the intein cleavage site and results in the purification of a target protein without any extra vector-derived residues at its C-terminus. After cleavage with SapI and NdeI the fragment was directed cloned into the expression vector PTYB1 that was cleaved in an analogous manner before. Introduction of the plasmids into E. coli JM109 for cloning and into E. coli ER2566 for expression and purification resulted in the mutant strains LG21 and LG31, respectively.

E. coli ER2566 containing pLGI1 used for heterologous expression of SugR was grown as a preculture $\mathrm{o} / \mathrm{N}$ in $10 \mathrm{ml}$ LB media with $100 \mu \mathrm{gml}^{-1}$ ampicillin at $37^{\circ} \mathrm{C}$. The main culture with a volume of $250 \mathrm{ml}$ was inoculated with a cell density of $0.1 \times 10^{8}$ cells $\times \mathrm{ml}^{-1}$ and grown to an o.D. of 0.5 to 0.8 at $37^{\circ} \mathrm{C}$. The expression of the proteins was acheived by IPTG $(0.5 \mathrm{mM})$ induction of the lac-promoter and lowering the culture temperature to $16^{\circ} \mathrm{C}$ in order to optimize T7 RNA-polymerase transcription. The culture was grown o/ $\mathrm{N}$ and all cells were pelleted by $6450 \times g$ for $15 \mathrm{~min}$ at $4^{\circ} \mathrm{C}$. The pellet was then resuspended in $25 \mathrm{ml}$ lysis buffer $\left(20 \mathrm{mM} \mathrm{Na} 2 \mathrm{HPO}_{4}, 500 \mathrm{mM} \mathrm{NaCl}, 1 \mathrm{mM}\right.$
EDTA, $0.1 \%$ TritonX-100, $20 \mu \mathrm{M}$ PMSF, $0.1 \mathrm{mM}$ TCEP, $\mathrm{pH} 8.0$ ) and the cells were disrupted by french press procedure at medium speed with 2000 psi repeating 3 times. The debris was pelleted at $6450 \times \mathrm{g}$ for $30 \mathrm{~min}$ at $4{ }^{\circ} \mathrm{C}$. The supernatant containing the crude protein extract was collected for IMPACT-CN purification (New England Biolabs, USA).

A $14 \mathrm{ml}$ Protino column (Macherey and Nagel, Germany) was prepared with $7 \mathrm{ml}$ of chitin beads and topped with a polycarbonate filter. Subsequently the packed column was equilibrated by washing with ten column volumes of precooled $\left(4^{\circ} \mathrm{C}\right)$ column buffer $\left(20 \mathrm{mM} \mathrm{Na}{ }_{2} \mathrm{HPO}_{4}, 500\right.$ $\mathrm{mM} \mathrm{NaCl}, 1$ mM EDTA, pH 8.0). Afterwards the column is ready for sample loading at $0.5-1 \mathrm{mlmin}^{-1}$. Again the column was washed with ten column volumes of column buffer which can be adjusted upto $1 \mathrm{M} \mathrm{NaCl}$. The cleavage of the intein is induced by washing with three column volumes of cleavage buffer $\left(20 \mathrm{mM} \mathrm{Na} \mathrm{HPO}_{4}, 500 \mathrm{mM}\right.$ $\mathrm{NaCl}, 1 \mathrm{mM}$ EDTA, $50 \mathrm{mM}$ DTT, pH 8.0), sealing the column to prevent drying and subsequent incubation at $4{ }^{\circ} \mathrm{C}$ for $16 \mathrm{~h}$. The elution of the recombinant protein can be performed by washing the column with 1-1.5 volumes of column buffer. In this case the elution volume of $7.5 \mathrm{ml}$ was applied to the column and the flowthrough was collected for the subsequent concentration step with Amicon Ultra-4 centrifugal filter device with a MW cutoff of $5 \mathrm{kDa}$ (Millipore, USA) down to an approximative volume of $250 \mu \mathrm{l}$. The concentrated elution fraction was washed 
twice with washing buffer $\left(20 \mathrm{mM} \mathrm{Na} \mathrm{HPO}_{4}, 10 \mathrm{mM}\right.$ $\mathrm{NaCl}, \mathrm{pH} 8.0$ ) again to a total volume of $250 \mu \mathrm{l}$. Different aliquots of the purification procedure can be collected (e.g. crude protein extract, flow through after the binding, and washing fractions) and tested with the purified protein by $1 \mathrm{D}-$ SDS-PAGE.

\section{Separation of cytoplasmic proteins of C. glutamicum RESI67}

One-dimensional denaturing sodium dodecyl sulfate polyacrylamide gel electroporesis (1D-SDS-PAGE) to separate proteins was used as described by Laemmli et al. [52] with a $4 \%$ stacking gel and a $12.5 \%$ resolving gel. Samples were denaturated in the presence of $2 \%$ SDS and $4 \%$ mercaptoethanol in Tris-HCl buffer $(60 \mathrm{mM}, \mathrm{pH} 6.8)$ by heating to $100^{\circ} \mathrm{C}$ for $5 \mathrm{~min}$. Apparent molecular weights were derived from the relative mobility of standard proteins as given in the Fermentas Protein Ladder (Fermentas Life Sciences $\mathrm{GmbH}$, St. Leon-Roth).

Subsequently SugR identification was obtained by peptide mass fingerprint analysis [53] utilizing the Bruker Ultraflex MALDI-TOF mass spectrometer (Bruker Daltonic, Bremen, Germany). Therefore, protein spots, which should be identified, were excised from the Coomassie stained gel and digested with a modified Trypsin enzyme (Promega, Mannheim, Germany). The protocol for tryptic digest and the settings of the MALDI-TOF-MS were described previously [52]. Peptide fingerprints thus obtained were compared with in silico generated tryptic fingerprints derived from the C. glutamicum ATCC 13032 genome data [17] by using the MASCOT software (MATRIX Science Ltd., London, UK; [54]). Best hits identified the corresponding genes of the proteins analyzed.

\section{Operator binding assays by electrophoretic mobility shift assay (EMSA) with purified SugR}

EMSA studies were performed with a set of Cy3-labeled PCR products, which were amplified using appropiate Cy3-labeled 20 mers (Operon, Germany). Unlabeled oligonucleotides representing putative binding sequences in front of $s u g R, p t s I / c g 2118$, and $p t s H$ were annealed with the corresponding complementary oligonucleotides under standard conditions [47]. The resulting doublestranded oligonucleotides were purified by means of Qiagen MinElute columns and used in EMSA displacement experiments.

During all EMSA studies 15 pmol of purified SugR protein was added to $4 \mu \mathrm{l}$ reaction buffer $\left(1 \mathrm{mM} \mathrm{MgCl}_{2}, 0.5 \mathrm{mM}\right.$ EDTA, $0.5 \mathrm{mM}$ DTT, $100 \mathrm{mM} \mathrm{NaCl}, 10 \mathrm{mM}$ Tris, $20 \%$ glycerin, $\mathrm{pH}$ 7.5). Subsequently, $3 \mu \mathrm{l} 87.9 \%$ glycerine, 0.05 pmol of Cy3-labeled PCR product and $\mathrm{H}_{2} \mathrm{O}$ was added to get a final volume of $15 \mu \mathrm{l}$. The assay was incubated at RT for 5 min and then separated with a $2 \%$ agar- ose gel prepared in gel buffer (40 mM Tris, $10 \mathrm{mM}$ sodium acetate, $1 \mathrm{mM}$ EDTA, pH7.8). A voltage of $14 \mathrm{Vcm}^{-1}$ was applied for $35 \mathrm{~min}$. The gel was then scanned with the Typhoon 8600 Variable Mode Imager (Amersham Biosciences Europe, Germany). During effector screening studies, the purified SugR protein was incubated at RT for 10 min with acetate, glucose, fructose, fructose-1-phosphate, fructose-1,6-bisphosphate, fructose-6-phosphate, or glucose-6-phosphate prior to the addition of the Cy3labeled PCR product. During EMSA competitory experiments, the purified SugR protein was added to reaction buffer, mixed with unlabeled competitor oligonucleotide and incubated at RT for 5 min prior to adding the labeled PCR fragments. After addition of the Cy3-labeled PCR product, the assay was incubated for additional $5 \mathrm{~min}$ before separating by gel electrophoresis.

\section{Total RNA Isolation from C. glutamicum cultures}

Cultures were grown to the logarithmic growth phase (o.D.600 = 10) and $1 \times 10^{9}$ cells were harvested by centrifugation at $16,000 \times g$ for $15 \mathrm{~s}$. The supernatant was removed by pipetting and the pellet immediately frozen in liquid nitrogen. The frozen cells were resuspended in $800 \mu$ l RLT-buffer (Rneasy Mini Kit, Qiagen) and instantly disrupted by means of the Ribolyser instrument (Hybaid, Heidelberg, Germany). Disruption was performed by three time intervals of $30 \mathrm{~s}$ at speed-level 6.5 with intermediary cooling of the probes on ice for $1 \mathrm{~min}$. Preparation of total RNA from C. glutamicum cells was performed as described by Hüser et al. [55].

\section{Determination of transcription starts by 5'-RACE}

Total RNA was isolated as described above. Primers binding downstream of the annotated translation starts of sugR, ptsI, cg2118, and ptsH were used along with $1.5 \mu \mathrm{g}$ of total RNA for cDNA synthesis. The cDNA was then modified and amplified using the 5'RACE Kit (Roche Diagnostics) according to the supplier's protocol. Resulting PCR products were ligated into the vector pCR2.1 by applying the TOPO TA cloning system and chemically competent E. coli TOP10 cells (Invitrogen). Sequencing of cloned RACE products was carried out by IIT Biotech (Bielefeld, Germany).

\section{Relative quantification of $m R N A$ levels using real-time $R T$ - PCR}

RT-PCR primers were designed to amplify an intergenic region of the analyzed gene of about 150-200 bp length with the Primer Design 4.2 software (Sci Ed Software) and were purchased from Operon (Qiagen, Germany). The real-time RT-PCR experiment was performed using the LightCycler instrument (Roche, Germany) in combination with the QuantiTect SYBR Green RT-PCR Kit (Qiagen, Germany) mixed with the specific primers and $300 \mathrm{ng}$ of sample RNA. 
The RT-PCR program consists of 3 segments starting with the reverse transcription at $50^{\circ} \mathrm{C}$ for $20 \mathrm{~min}$, followed by the initial activation of the HotStar Taq DNA polymerase at $95^{\circ} \mathrm{C}$ for $15 \mathrm{~min}$. Thirdly the RT-PCR step was performed 55 times in cycles of: $95^{\circ} \mathrm{C}$ for $15 \mathrm{~s}, 55^{\circ} \mathrm{C}$ for $30 \mathrm{~s}$, and $72^{\circ} \mathrm{C}$ for 20s. The melting curve was recorded over a range of 65 to $95^{\circ} \mathrm{C}$ with a heating slope of $0.1^{\circ} \mathrm{C}$ pers at continuous fluorescence measurement. The crossing point for each gene and condition was calculated by the data analysis method provided by the LightCycler software, using the maximum increase or acceleration of fluorescence.

\section{Microarray experiments and analysis}

Total RNA isolated from C. glutamicum was also used for global transcriptional analyses. The cDNA synthesis and array hybridization were performed as described by Hüser et al. [55]. Data analysis was performed with the ImaGene V6.0 and EMMA V2.2 [56] software packages. Evaluation of the hybridization experiment was done as described by Rey et al. [57] using an $m$-value cutoff of \pm 1 , which corresponds to expression changes equal or greater than 2 fold. Furthermore, $m$-values were considered as significant if the Student's t-test resulted in a $p$-value $\leq 0.05$. The microarrays used represent all 3002 coding regions of $C$. glutamicum RES167 as 70 mer oligonucleotides.

\section{Bioinformatic tools used to analyse nucleotide sequences}

For interpreting the data of the C. glutamicum ATCC 13032 genome project the automated sequence investigation program GenDB [58] was used. Sequence comparisons were carried out by using the ClustalX software [59].

\section{Authors' contributions}

LG carried out the experimental work and drafted the manuscript. JPS participated during the microarray analyses. MH constructed the cg2118 deletion mutant. SM participated during experimental work and illustration design. AT participated in supervision. AP aided in coordination and participated in supervision. JK conceived of the study and participated in coordination and supervision. All authors read and approved the final manuscript.

\section{Acknowledgements}

We like to thank Dr. Andrea Hüser for providing the microarray technique and support by conducting the microarray hybridization as well as the Bundesministerium für Bildung und Forschung for financial support.

\section{References}

I. Postma PW, Lengeler JW, Jacobson GR: Phosphoenolpyruvate:carbohydrate phosphotransferase systems of bacteria. Microbiol Rev 1993, 57(3):543-594.

2. Deutscher J, Francke C, Postma PW: How phosphotransferase system-related protein phosphorylation regulates carbohydrate metabolism in bacteria. Microbiol Mol Biol Rev 2006, 70(4):939-103I.

3. Robillard GT, Broos ]: Structure/function studies on the bacterial carbohydrate transporters, enzymes II, of the phosphoe- nolpyruvate-dependent phosphotransferase system. Biochim Biophys Acta 1999, I 422(2):73-104.

4. Hermann T: Industrial production of amino acids by coryneform bacteria. Journal of biotechnology 2003, 104(I-3): 155- 172.

5. Malin GM, Bourd GI: Phosphotransferase-dependent glucose transport in Corynebacterium glutamicum. Journal of Applied Bacteriology |991, 71:5 17-523.

6. Dominguez H, Lindley ND: Complete Sucrose Metabolism Requires Fructose Phosphotransferase Activity in Corynebacterium glutamicum. To Ensure Phosphorylation of Liberated Fructose. Appl Environ Micorbiol 1996, 62(10):3878-3880.

7. Parche S, Burkovski A, Sprenger GA, Weil B, Kramer R, Titgemeyer F: Corynebacterium glutamicum: a dissection of the PTS. J Mol Microbiol Biotechnol 200I, 3(3):423-428.

8. Moon M, Kim H, Oh T, Shin C, Lee J, Kim S, Lee J: Analyses of enzyme II gene mutants for sugar transport and heterologous expression of fructokinase gene in Corynebacterium glutamicum. FEMS Microbiology Letters 2005, 244:259-266.

9. Titgemeyer F, Hillen W: Global control of sugar metabolism: a gram-positive solution. Antonie Van Leeuwenhoek 2002, 82(I-4):59-7I.

10. Lee JK, Sung MH, Yoon KH, Yu JH, Oh TK: Nucleotide sequence of the gene encoding the Corynebacterium glutamicum mannose enzyme II and analyses of the deduced protein sequence. FEMS microbiology letters 1994, I I 9(1-2): I 37- 45 .

II. Mori M, Shiio I: Phosphenolpyruvate: Sugar phosphotransferase systems and sugar metabolism in Brevibacterium flavum. Agr Biol Chem 1987, 5 I:2671-2678.

12. Ryu S, Ramseier TM, Michotey V, Saier MH Jr, Garges S: Effect of the FruR regulator on transcription of the pts operon in Escherichia coli. J Biol Chem 1995, 270(6):2489-2496.

13. Barriere C, Veiga-da-Cunha M, Pons N, Guedon E, van Hijum SA, Kok J, Kuipers OP, Ehrlich DS, Renault P: Fructose utilization in Lactococcus lactis as a model for low-GC gram-positive bacteria: its regulator, signal, and DNA-binding site. J Bacteriol 2005, I87(II):3752-376I.

14. Gaurivaud P, Laigret F, Verdin E, Garnier M, Bove JM: Fructose operon mutants of Spiroplasma citri. Microbiology 2000, I46(Pt 9):2229-2236.

15. Loo CY, Mitrakul K, Voss IB, Hughes CV, Ganeshkumar N: Involvement of an inducible fructose phosphotransferase operon in Streptococcus gordonii biofilm formation. J Bacteriol 2003, I 85(2I):624I-6254.

16. Parche S, Thomae AW, Schlicht M, Titgemeyer F: Corynebacterium diphtheriae: a PTS view to the genome. J Mol Microbiol Biotechnol 200I, 3(3):4I5-422.

17. Kalinowski J, Bathe B, Bartels D, Bischoff N, Bott M, Burkovski A, Dusch N, Eggeling L, Eikmanns BJ, Gaigalat L, Goesmann A, Hartmann M, Huthmacher K, Kramer R, Linke B, McHardy AC, Meyer F, Mockel B, Pfefferle W, Pühler A, Rey DA, Rückert C, Rupp O, Sahm H, Wendisch VF, Wiegrabe I, Tauch A: The complete Corynebacterium glutamicum ATCC 13032 genome sequence and its impact on the production of L-aspartate-derived amino acids and vitamins. J Biotechnol 2003, I04(I-3):5-25.

18. Kotrba $P$, Inui M, Yukawa H: A single V3 I 7 A or V3 I 7 M substitution in Enzyme II of a newly identified beta-glucoside phosphotransferase and utilization system of Corynebacterium glutamicum $\mathbf{R}$ extends its specificity towards cellobiose. Microbiology (Reading, England) 2003, I 49(Pt 6): 1569-1580.

19. Dominguez H, Rollin C, Guyonvarch A, Guerquin-Kern JL, CocaignBousquet M, Lindley ND: Carbon-flux distribution in the central metabolic pathways of Corynebacterium glutamicum during growth on fructose. Eur J Biochem 1998, 254(I):96-102.

20. Krömer JO, Sorgenfrei O, Klopprogge K, Heinzle E, Wittmann C: InDepth Profiling of Lysine-Producing Corynebacterium glutamicum by Combined Analysis of the Transcriptome, Metabolome, and Fluxome. Journal of Bacteriology 2003, 186(6): | 769-1784.

21. Moon MW, Park SY, Choi SK, Lee JK: The phosphotransferase system of Corynebacterium glutamicum: features of sugar transport and carbon regulation. J Mol Microbiol Biotechnol 2007, I 2(I-2):43-50.

22. Engels V, Wendisch V: The DeoR-type regulator SugR represses expression of ptsG in Corynebacterium glutamicum. J Bacteriol 2007, I 89(8):2955-2966. 
23. Lee SJ, Moulakakis C, Koning SM, Hausner W, Thomm M, Boos W: TrmB, a sugar sensing regulator of $A B C$ transporter genes in Pyrococcus furiosus, exhibits dual promoter specificity and is controlled by different inducers. Mol Microbiol 2005, 57(6): $1797-1807$

24. Lee S], Surma M, Seitz S, Hausner W, Thomm M, Boos W: Differential signal transduction via TrmB, a sugar sensing transcriptional repressor of Pyrococcus furiosus. Mol Microbiol 2007, 64(6): | 499-I505.

25. Price MN, Huang KH, Alm EJ, Arkin AP: A novel method for accurate operon predictions in all sequenced prokaryotes. Nucleic Acids Res 2005, 33(3):880-892.

26. Ermolaeva MD, Khalak HG, White O, Smith HO, Salzberg SL: Prediction of transcription terminators in bacterial genomes. Mol Biol 2000, 30 I (I):27-33.

27. Patek M, Nesvera J, Guyonvarch A, Reyes O, Leblon G: Promoters of Corynebacterium glutamicum. Journal of Biotechnology 2003, I 04(I-3):3I I-323.

28. Brune I, Jochmann N, Brinkrolf K, Hüser AT, Gerstmeir R, Eikmanns B], Kalinowski J, Pühler A, Tauch A: The IcIR-type transcriptional repressor LtbR regulates the expression of leucine and tryptophan biosynthesis genes in the amino acid producer Corynebacterium glutamicum. I Bacteriol 2007, I 89(7):2720-33.

29. Chong S, Montello GE, Zhang A, Cantor EJ, Liao W, Xu MQ, Benner $\mathrm{J}$ : Utilizing the $\mathrm{C}$-terminal cleavage activity of a protein splicing element to purify recombinant proteins in a single chromatographic step. Nucleic Acids Res I998, 26(22):5I09-5 I I 5.

30. van Rooijen RJ, de Vos WM: Molecular cloning, transcriptional analysis, and nucleotide sequence of lacR, a gene encoding the repressor of the lactose phosphotransferase system of Lactococcus lactis. J Biol Chem 1990, 265(30): I8499-I8503.

31. Oskouian B, Stewart GC: Repression and catabolite repression of the lactose operon of Staphylococcus aureus. J Bacteriol 1990 I 72(7):3804-38I2.

32. Yebra MJ, Veyrat A, Santos MA, Perez-Martinez G: Genetics of Lsorbose transport and metabolism in Lactobacillus casei. Bacteriol 2000, I 82(I): |55-163.

33. Saxild HH, Andersen LN, Hammer K: Dra-nupC-pdp operon of Bacillus subtilis: nucleotide sequence, induction by deoxyribonucleosides, and transcriptional regulation by the deoRencoded DeoR repressor protein. J Bacteriol 1996, I 78(2):424-434.

34. Valentin-Hansen P, Albrechtsen B, Love Larsen JE: DNA-protein recognition: demonstration of three genetically separated operator elements that are required for repression of the Escherichia coli deoCABD promoters by the DeoR repressor. Embo J 1986, 5(8):2015-2021.

35. Christensen M, Borza T, Dandanell G, Gilles AM, Barzu O, Kelln RA Neuhard J: Regulation of expression of the 2-deoxy-D-ribose utilization regulon, deoQKPX, from Salmonella enterica serovar typhimurium. J Bacteriol 2003, I 85(20):6042-6050.

36. Elgrably-Weiss M, Schlosser-Silverman E, Rosenshine I, Altuvia S: DeoT, a DeoR-type transcriptional regulator of multiple target genes. FEMS Microbiol Lett 2006, 254(I): |4|-|48.

37. Zeng X, Saxild HH, Switzer RL: Purification and characterization of the DeoR repressor of Bacillus subtilis. Journal of bacteriology 2000, I 82(7):1916-22

38. Babu MM, Teichmann SA: Functional determinants of transcription factors in Escherichia coli: protein families and binding sites. Trends in Genetics 2003, I 9(2):75-79.

39. Dandanell G, Valentin-Hansen P, Larsen JE, Hammer K: Long-range cooperativity between gene regulatory sequences in $a$ prokaryote. Nature 1987, 325(6 1 07):823-826.

40. Ramseier TM, Negre D, Cortay JC, Scarabel M, Cozzone AJ, Saier MH Jr: In vitro binding of the pleiotropic transcriptional regulatory protein, FruR, to the fru, pps, ace, pts and icd operons of Escherichia coli and Salmonella typhimurium. J Mol Biol 1993, 234(I):28-44

4I. Saier MH Jr, Ramseier TM: The catabolite repressor/activator (Cra) protein of enteric bacteria. Journal of bacteriology 1996, | 78(12):34| |-34|7.

42. Georgi T, Rittmann D, Wendisch VF: Lysine and glutamate production by Corynebacterium glutamicum on glucose, fructose and sucrose: roles of malic enzyme and fructose- I,6-bisphosphate. Metabolic Engineering 2005, 7:291-301.
43. Gerstmeir R, Wendisch VF, Schnicke S, Ruan H, Farwick M, Reinscheid D, Eikmanns BJ: Acetate metabolism and its regulation in Corynebacterium glutamicum. Journal of biotechnology 2003, I 04(I-3):99-122.

44. Gerstmeir R, Cramer A, Dangel P, Schaffer S, Eikmanns BJ: RamB, a novel transcriptional regulator of genes involved in acetate metabolism of Corynebacterium glutamicum. J Bacetriol 2004, I 86(9):2798-2809

45. Sambrook J, Fritsch EF, Maniatis T: Molecular Cloning: A Laboratory Manual. Cold Spring Harbor. NY, USA: Cold Spring Harbor Laboratory Press:; 1989.

46. Suggs SV, Hirose T, Miyake T, Kawashima EH, Johnson MJ, Itakura K Wallace RB: Use of synthetic oligo deoxyribonucleotides for the isolation of specific cloned DNA sequences. Edited by: Brown DD, Fox CF. Developemental biology using purified genes Academic Press, New York; 1981:683-693.

47. Tauch A, Kirchner O, Löffler B, Götker S, Pühler A, Kalinowski J: Efficient electrotransformation of Corynebacterium diphtheriae with a mini-replicon derived from the Corynebacterium glutamicum plasmid pGAI. Curr Microbiol 2002, 45(5):362-367.

48. Haynes JA, Britz ML: Electrotransformation of Brevibacterium lactofermentum and Corynebacterium glutamicum: growth in Tween $\mathbf{8 0}$ increases transformation frequencies. FEMS Microbiol Letters 1989, 6 I:329-334.

49. Horton RM: PCR-mediated recombination and mutagenesis. SOEing together tailor-made genes. Mol Biotechnol 1995, 3(2):93-99.

50. Schäfer A, Tauch A, Jager W, Kalinowski J, Thierbach G, Pühler A: Small mobilizable multi-purpose cloning vectors derived from the Escherichia coli plasmids pK I 8 and pKI9: selection of defined deletions in the chromosome of Corynebacterium glutamicum. Gene 1994, I45(I):69-73.

5I. Rückert C, Pühler A, Kalinowski J: Genome-wide analysis of the L-methionine biosynthetic pathway in Corynebacterium glutamicum by targeted gene deletion and homologous complementation. J Biotechnol 2003, I 04(I-3):2 I 3-228.

52. Laemmli UK: Cleavage of structural proteins during the assembly of the head of bacteriophage T4. Nature 1970, 227(5259):680-685.

53. Henzel W], Billeci TM, Stults IT, Wong SC, Grimley C, Watanabe C: Identifying proteins from two-dimensional gels by molecular mass searching of peptide fragments in protein sequence databases. Proc Natl Acad Sci USA 1993, 90( I I):50 I I-50 I5.

54. Perkins DN, Pappin DJ, Creasy DM, Cottrell JS: Probability-based protein identification by searching sequence databases using mass spectrometry data. Electrophoresis 1999, 20( I 8):355|-3567.

55. Hüser AT, Becker A, Brune I, Dondrup M, Kalinowski J, Plassmeier J, Pühler A, Wiegräbe I, Tauch A: Development of a Corynebacterium glutamicum DNA microarray and validation by genomewide expression profiling during growth with propionate as carbon source. J Biotechnol 2003, I06(2-3):269-286.

56. Dondrup M, Goesmann A, Bartels D, Kalinowski J, Krause L, Linke B, Rupp O, Sczyrba A, Pühler A, Meyer F: EMMA: a platform for consistent storage and efficient analysis of microarray data. Journal of biotechnology 2003, 106(2-3):135-146.

57. Rey DA, Nentwich SS, Koch DJ, Rückert C, Pühler A, Tauch A, Kalinowski J: The McbR repressor modulated by the effector substance $\mathrm{S}$-adenosylhomocysteine controls directly the transcription of a regulon involved in sulphur metabolism of Corynebacterium glutamicum ATCC 13032. Mol Microbiol 2005, 56(4):87|-887

58. Meyer F, Goesmann A, McHardy AC, Bartels D, Bekel T, Clausen J, Kalinowski J, Linke B, Rupp O, Giegerich R, Pühler A: GenDB-an open source genome annotation system for prokaryote genomes. Nucleic Acids Res 2003, 3 I (8):2 187-2195.

59. Thompson JD, Gibson TJ, Plewniak F, Jeanmougin F, Higgins DG: The CLUSTAL_X windows interface: flexible strategies for multiple sequence alignment aided by quality analysis tools. Nucleic Acids Res 1997, 25(24):4876-4882 